\title{
CDKL5 ensures excitatory synapse stability by reinforcing NGL-1-PSD95 interaction in the postsynaptic compartment and is impaired in patient iPSC-derived neurons
}

Sara Ricciardi ${ }^{1}$, Federica Ungaro ${ }^{1,7}$, Melanie Hambrock ${ }^{2,7}$, Nils Rademacher ${ }^{2}$, Gilda Stefanelli ${ }^{1}$, Dario Brambilla ${ }^{3}$, Alessandro Sessa ${ }^{1}$, Cinzia Magagnotti ${ }^{4}$, Angela Bachi ${ }^{4}$, Elisa Giarda ${ }^{5}$, Chiara Verpelli ${ }^{6}$, Charlotte Kilstrup-Nielsen ${ }^{5}$, Carlo Sala ${ }^{6}$, Vera M. Kalscheuer ${ }^{2,8,9}$ and Vania Broccoli ${ }^{1,8,9}$

Mutations of the cyclin-dependent kinase-like 5 (CDKL5) and netrin-G1 (NTNG1) genes cause a severe neurodevelopmental disorder with clinical features that are closely related to Rett syndrome, including intellectual disability, early-onset intractable epilepsy and autism. We report here that CDKL5 is localized at excitatory synapses and contributes to correct dendritic spine structure and synapse activity. To exert this role, CDKL5 binds and phosphorylates the cell adhesion molecule NGL-1. This phosphorylation event ensures a stable association between NGL-1 and PSD95. Accordingly, phospho-mutant NGL-1 is unable to induce synaptic contacts whereas its phospho-mimetic form binds PSD95 more efficiently and partially rescues the CDKL5-specific spine defects. Interestingly, similarly to rodent neurons, iPSC-derived neurons from patients with CDKL5 mutations exhibit aberrant dendritic spines, thus suggesting a common function of CDKL5 in mice and humans.

Rett syndrome (RTT) is a severe neurodevelopmental disorder and is the most common genetic cause of intellectual disability in females ${ }^{1,2}$. The disorder is mainly caused by mutations in the methyl-CpG-binding protein 2 (MECP2) gene $\mathrm{e}^{3,4}$. In addition to classical RTT, several atypical forms exist, including the Hanefeld variant, which is caused by mutations in the X-linked CDKL5 gene. Patients present with early-onset intractable seizures starting before the age of 6 months and also show some typical symptoms of RTT although the clinical spectrum is heterogeneous ${ }^{5-11}$. This suggests that CDKL5 mutations cause an early-onset neurodevelopmental disorder ${ }^{12}$. In a small number of patients, CDKL5 deletions have been reported ${ }^{13-15}$. These very probably caused loss of CDKL5 function, similarly to what has previously been found in patients with a chromosome translocation that disrupted CDKL5 (refs 8,9). CDKL5 encodes a serine/threonine kinase whose catalytic domain shares homology with members of the cyclin-dependent kinase family and mitogen-activated protein kinases ${ }^{16,17}$. Interestingly, we showed that CDKL5 and MeCP2 are both induced at high levels during neuronal maturation and synaptogenesis. In addition, CDKL5 binds and phosphorylates MeCP2 in vitro ${ }^{18}$. Despite this molecular interplay, it is now well established that CDKL5 exerts its own functions, regardless of MeCP2 (refs 19-22). However, the functional role of CDKL5 is unknown at present and, thus, the question of how CDKL5 mutations affect neuronal development and function and contribute to the pathophysiology of RTT remains to be answered.

Herein, we describe that CDKL5 silencing leads to severe deficits in spine density and morphology. Notably, similar alterations were found in neurons established from patient fibroblast-derived pluripotent stem cells (iPSCs). Furthermore, we identified the netrin-G1 ligand (NGL-1, also known as LRRC4C) as a direct interactor and substrate of CDKL5. Importantly, NGL-1 phosphorylation strengthens the NGL-1-PSD95 interaction. Our findings demonstrate a role for CDKL5 in spine development and synapse morphogenesis.

${ }^{1}$ Stem Cell and Neurogenesis Unit, Division of Neuroscience, San Raffaele Scientific Institute, Via Olgettina 58, 20132 Milan, Italy. ${ }^{2}$ Max Planck Institute for

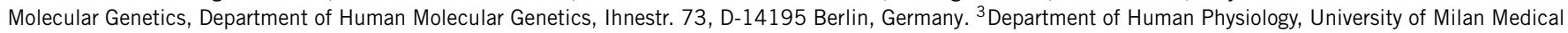

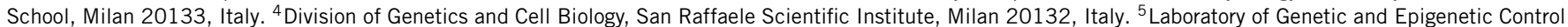
of Gene Expression, Department of Structural and Functional Biology, University of Insubria, 21052 Busto Arsizio, VA, Italy. ${ }^{6}$ Consiglio Nazionale delle Ricerche Neuroscience Institute, Milan 20129, Italy. ${ }^{7}$ These authors contributed equally to this work. ${ }^{8}$ Equally contributing senior authors.

${ }^{9}$ Correspondence should be addressed to V.M.K. or V.B. (e-mail: kalscheu@molgen.mpg.de or broccoli.vania@hsr.it) 
a

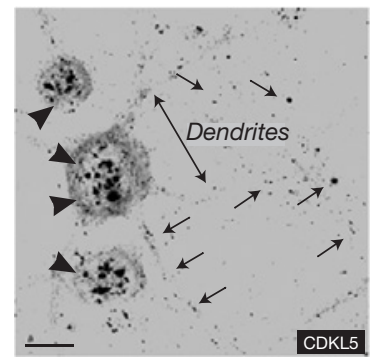

b

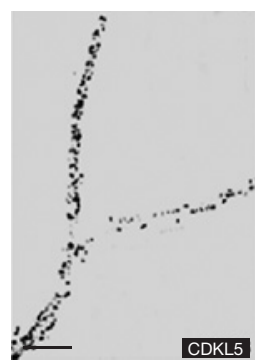

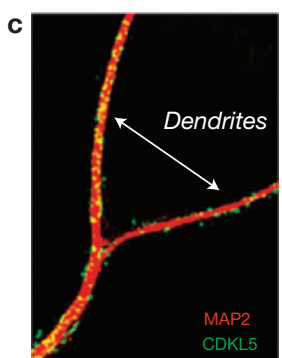

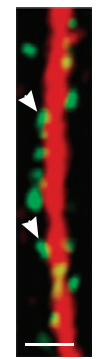

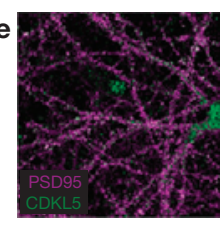

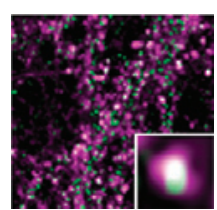

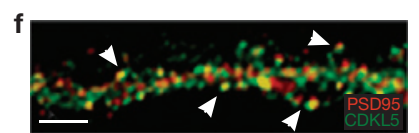

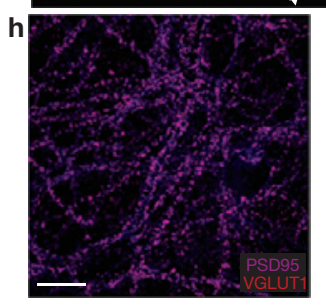

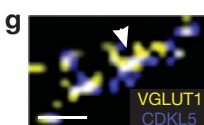
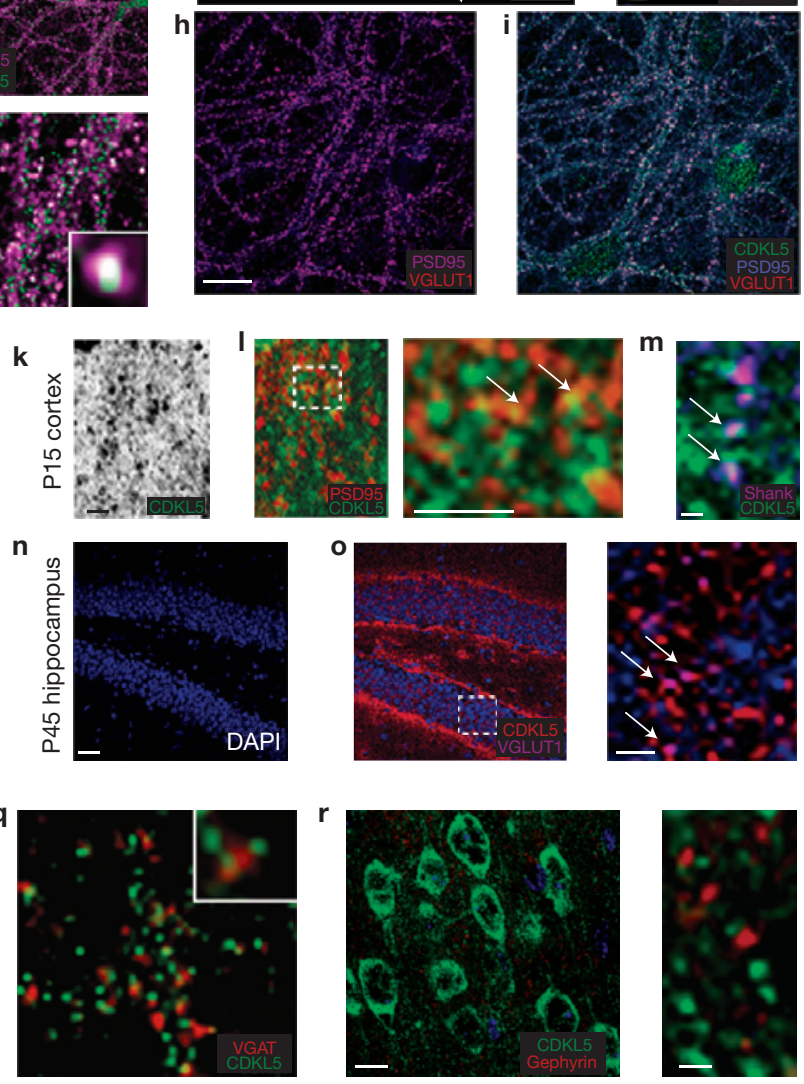
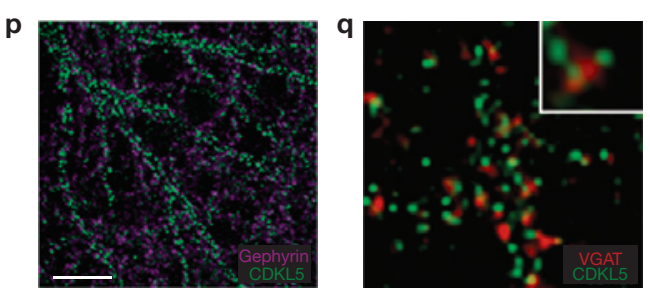

Figure 1 CDKL5 localizes in dendrites and gathers at excitatory synapses both in vitro and in vivo. (a) Primary hippocampal neurons immunolabelled at DIV15 with an antibody against CDKL5. The arrowheads point to intranuclear CDKL5 immunolocalization. The arrows point to CDKL5 immunolabelling in neuronal dendrites. (b,c) Primary hippocampal neurons at DIV15 co-immunostained for CDKL5 and MAP2. The right panel in c shows a higher magnification of the left panel. The arrowheads point to CDKL5 dendritic puncta localized to dendritic spines. (d-f) Primary hippocampal neurons immunolabelled at DIV15 with antibodies against CDKL5 and PSD95. The bottom panels in $\mathbf{d}$ and e show higher magnifications of the top panels. The inset in the bottom panel of $\mathbf{e}$ and the arrowheads in $\mathbf{f}$ show domains of co-localization between CDKL5 and PSD95. (g) Immunostaining of hippocampal neurons with CDKL5 and VGLUT1. The arrowhead indicates co-localization of CDKL5 and VGLUT1. (h,i) Immunostaining of CDKL5 in hippocampal neurons at DIV15 shows CDKL5 clustering at excitatory synapses co-localizing with PSD95 and apposed to VGLUT1. (j) Quantification of the mean percentage of co-localization ( \pm s.e.m.) of endogenous CDKL5 with PSD95, Shank, NR2 and VGLUT1. $n=10$ neurons for each. $n$ is derived from three experiments. (k-o) Immunolocalization of CDKL5 in mouse brain also shows CDKL5 clustering at excitatory synapses, as shown by apposition with PSD95 (I) or Shank (m) in P15 mouse cortex and with VGLUT1 in P45 mouse hippocampus ( $\mathbf{n}-\mathbf{0})$. The right panels in I and $\mathbf{o}$ show a higher magnification of area outlined in the left panel. The arrows in I (right), $\mathbf{m}$ and $\mathbf{o}$ (right) point to a region of co-localization of CDKL5 with either PSD95, Shank or VGLUT1. (p-r) Immunostaining with CDKL5 and either gephyrin or VGAT antibodies both in vitro $(\mathbf{p}, \mathbf{q})$ and in vivo (r). The inset in $\mathbf{q}$ and the right panel of $\mathbf{r}$ highlight separate protein domains between CDKL5 and VGAT and Gephyrin, respectively. (s) Quantification of the mean percentage of co-localization ( \pm s.e.m.) of endogenous CDKL5 with gephyrin and VGAT. $n=10$ neurons for each. $n$ is derived from three experiments. (t) CDKL5 is detected in the synaptosomal fraction (Syp) and is enriched in PSD fraction I (PSDI). Note that CDKL5 is also detected in PSD fractions II and III (PSDII and PSDIII). PSD95 and synaptophysin (Syn) were used as a control. 1-Triton/2-Triton represents extraction with Triton X-100 once/twice. Scale bars, $100 \mu \mathrm{m}$ (n); $10 \mu \mathrm{m}$ (a, b, d (top), h, k, p, r (left)); $5 \mu \mathrm{m}$ (d (bottom), f, I (right), o (right)); $3 \mu \mathrm{m}$ (c (right), g, m); $1 \mu \mathrm{m}$ (r (right)). Uncropped images of blots are shown in Supplementary Fig. S8. 


\section{RESULTS}

\section{CDKL5 is enriched at the PSD of glutamatergic synapses}

In the mouse, CDKL5 levels were highest in brain (Supplementary Fig. S1a,b). In postnatal day (P) 21 brain, CDKL5 immunoreactivity was evident in a punctate pattern in cell bodies as previously described $^{20}$ (Fig. 1a), and also along dendrites (Fig. 1b,c). The expression of CDKL5 steadily increased during early postnatal brain development and, likewise, during maturation of in vitro cultured neurons (Supplementary Fig. S1c-f). Interestingly, some of the CDKL5 dendritic puncta localized to dendritic spines (Fig. 1c, right). Subsequently, we investigated whether CDKL5 is present at the postsynaptic density (PSD) and found that a large number of CDKL5 puncta $(61 \pm 3 \%)$ co-localized with PSD95 in neurons at 15 days in vitro (DIV15; Fig. 1d-f). Likewise, CDKL5 staining closely matched the pattern of other PSD markers (Fig. 1j). Consistent with a postsynaptic localization, CDKL5 immunolabelling was closely juxtaposed with presynaptic VGLUT1 (Fig. 1g). Triple staining for CDKL5, PSD95 and VGLUT1 confirmed CDKL5 localization at excitatory synapses (Fig. 1h,i). CDKL5 puncta coincided with the dot-like immunostaining of PSD95 and SHANK (Fig. 1k-m) and were apposed to VGLUT1 puncta also in brain (Fig. 1n,o). To confirm the presence of CDKL5 at the PSD, we performed a subcellular fractionation of mouse brain. CDKL5 was included in the synaptic fraction and in the entire PSD fraction (Fig. 1t). Further detergent solubilization of the synaptic plasma membrane fraction showed that CDKL5 is detectable in all PSD fractions, indicating its association with the PSD (Fig. 1t). Our studies also showed that CDKL5 co-localized marginally with inhibitory synaptic markers (Fig. 1p-s). Taken together, these findings indicate that CDKL5 is almost exclusively localized at excitatory synapses both in vitro and in vivo.

\section{Loss of CDKL5 impairs spine structure and synaptic activity}

To investigate the role of CDKL5 in dendritic spines, we silenced CDKL5 in hippocampal neurons using two short-hairpin RNAs (shRNAs; sh-CDKL5\#1 and sh-CDKL5\#2). In HEK293T cells, both shRNAs downregulated exogenous mouse CDKL5 levels by almost $80 \%$ (Supplementary Fig. S2a). Next, we infected DIV7 hippocampal neurons with sh-CDKL5\#1 and seven days later observed a consistent reduction in the level of CDKL5 (Supplementary Fig. S2b-h). Knockdown neurons showed a significant increase in protrusion density (Fig. 2a-i), and the dendritic protrusions were significantly thinner when compared with controls and showed a filopodialike morphology (Fig. 2a-i). Notably, some of these thin filopodialike spines were particularly branched and exhibited an aberrant morphology (middle and bottom panel in the top right of Fig. 2b). Furthermore, the percentage of filopodia-like spines and thin-headed spines increased, whereas the percentage of stubby and mushroomshaped spines decreased (Fig. 2h,i). We obtained similar results for neurons infected at DIV10 and analysed at DIV15 (data not shown). Conversely, we did not score any significant alteration in dendritic arborization (data not shown). These results indicate that CDKL5 is required for ensuring a correct number of well-shaped spines. Specificity was confirmed by the ability of a CDKL5 knockdown resistant form to rescue both normal spine morphology and density (Supplementary Fig. S2i-o). The morphological alterations reported in CDKL5-silenced neurons were associated with a reduction in the number of excitatory synapses and synaptophysin puncta in these neurons (Fig. $2 \mathrm{j}-\mathrm{s}$ ). To determine whether these alterations were accompanied by a reduction in the overall level of synaptic activity, we examined spontaneous miniature excitatory postsynaptic currents (mEPSCs) in sh-CDKL5\#1-transfected neurons. Consistent with the reduced excitatory synapse density, CDKL5 knockdown caused a significant decrease of mEPSCs (Fig. 2t-y) but had no significant effect on inhibitory synapse density and accordingly we did not detect any significant changes in miniature inhibitory postsynaptic currents (Supplementary Fig. S3). These results indicate that CDKL5 is necessary for correct dendritic spine structure and synapse activity.

Considering that CDKL5 is a kinase, we reasoned that its phosphorylation activity is necessary for this function. To address this issue we used the CDKL $5^{\mathrm{K} 42 \mathrm{R}}$ kinase-dead form, which has a dominant-negative effect on wild-type CDKL5 (refs 16,19). To analyse the effects of CDKL5 $5^{\mathrm{K} 42 \mathrm{R}}$ on spine density and morphology, we transfected DIV7 hippocampal neurons with DsRed alone or combined with GFP-CDKL5 ${ }^{\mathrm{K} 42 \mathrm{R}}$. Interestingly, GFP-CDKL5 ${ }^{\mathrm{K} 42 \mathrm{R}}$ immunolabelling was very similar to that of endogenous CDKL5 and there was a clear enrichment of the immunostaining in dendritic spines (Supplementary Fig. S2p- $\mathrm{q}^{\prime}$ ). Consistent with its dominant-negative action, neurons overexpressing GFP-CDKL5 ${ }^{\mathrm{K} 42 \mathrm{R}}$ exhibited a significantly higher number of thinner and longer dendritic protrusions and a corresponding loss of stubby and mushroom-shaped spines with respect to controls (Supplementary Fig. S2r-w). As the phenotype discovered for the CDKL5 ${ }^{\mathrm{K} 42 \mathrm{R}}$ mutant was similar to the phenotype of CDKL5knockdown neurons, it is conceivable that CDKL5 promotes spine formation through a mechanism that is dependent on its kinase activity.

Next, we sought to confirm the significance of our findings in vivo by electroporating embryonic day (E) 13.5 embryos in utero with a plasmid containing cassettes for independent expression of sh-CDKL5\#1 and EGFP. Interestingly, at E18.5, CDKL5-downregulated neurons showed a delay in radial migration. On the contrary, at P11 their relative location within the cerebral cortex was comparable to that of controls, thus indicating a transient delay in the definitive neuronal positioning (Fig. 3a,b and data not shown). Imaging of the GFP-labelled dendritic protrusions at P11, a crucial period of synaptogenesis, showed numerous protrusions with a large fraction having a well-defined head structure, characteristic of mature spines. In agreement with the in vitro studies, sh-CDKL5\#1-knockdown cortical pyramidal neurons showed a robust increase in the protrusion density with significantly longer and dysmorphic dendritic protrusions (Fig. 3c-h).

Next, we examined whether CDKL5 deficit might affect the number of functional excitatory synapses also in vivo. The density of VGLUT1 puncta identifying excitatory presynaptic buttons was significantly lower in sh-CDKL5\#1 than in sh-control pyramidal neurons (Fig. 3e,f,i), confirming that CDKL5 is crucial for dendritic spine morphogenesis and synaptic contact maintenance in vivo.

\section{NGL cell adhesion molecules interact with CDKL5 at the PSD}

Next, we set out to identify new CDKL5-interacting partners within dendritic spines. On the basis of our previous results showing that truncation of NTNG1 caused atypical RTT with early-onset seizures ${ }^{23}$, we considered the netrin-G1 receptor NGL-1 and the other members of the NGL family (NGL-2 and -3, also known as LRRC4 and LRRC4B), which play a crucial role in early synapse formation and subsequent 

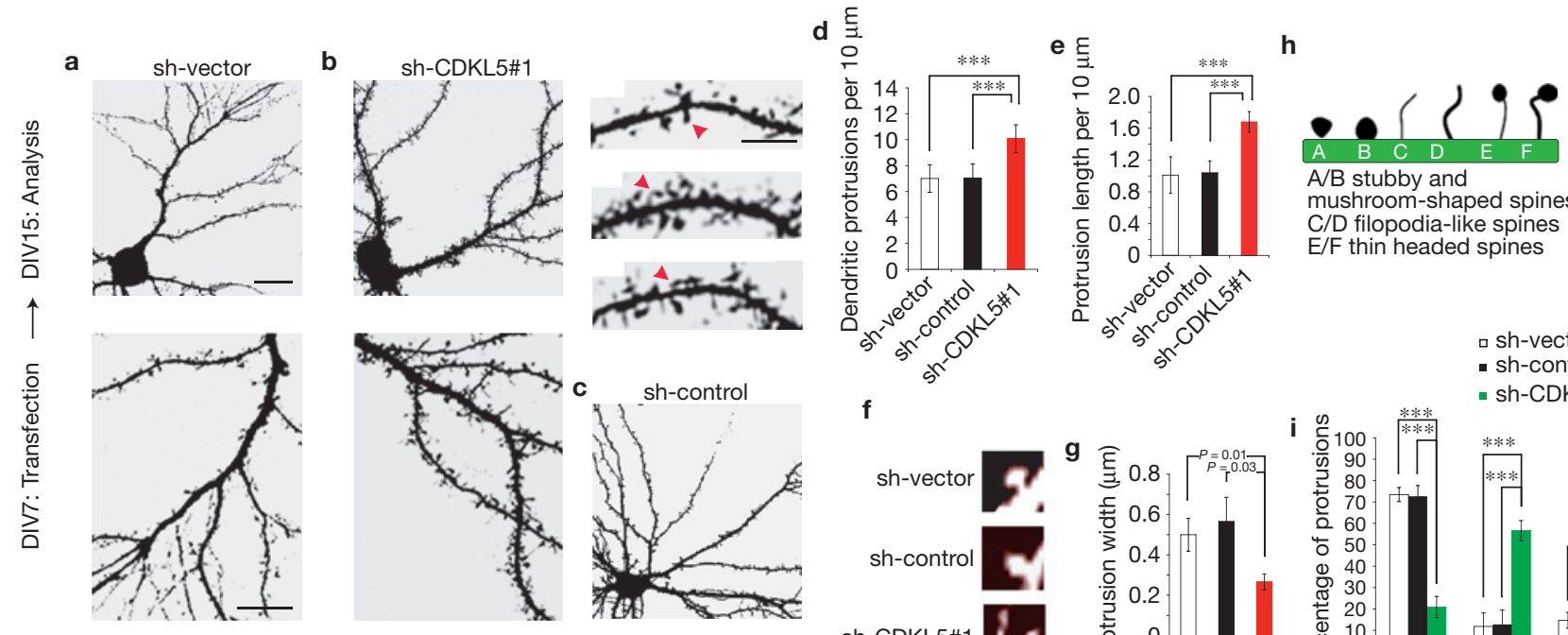

A/B stubby and

mushroom-shaped spines

C/D filopodia-like spines
E/F thin headed spines
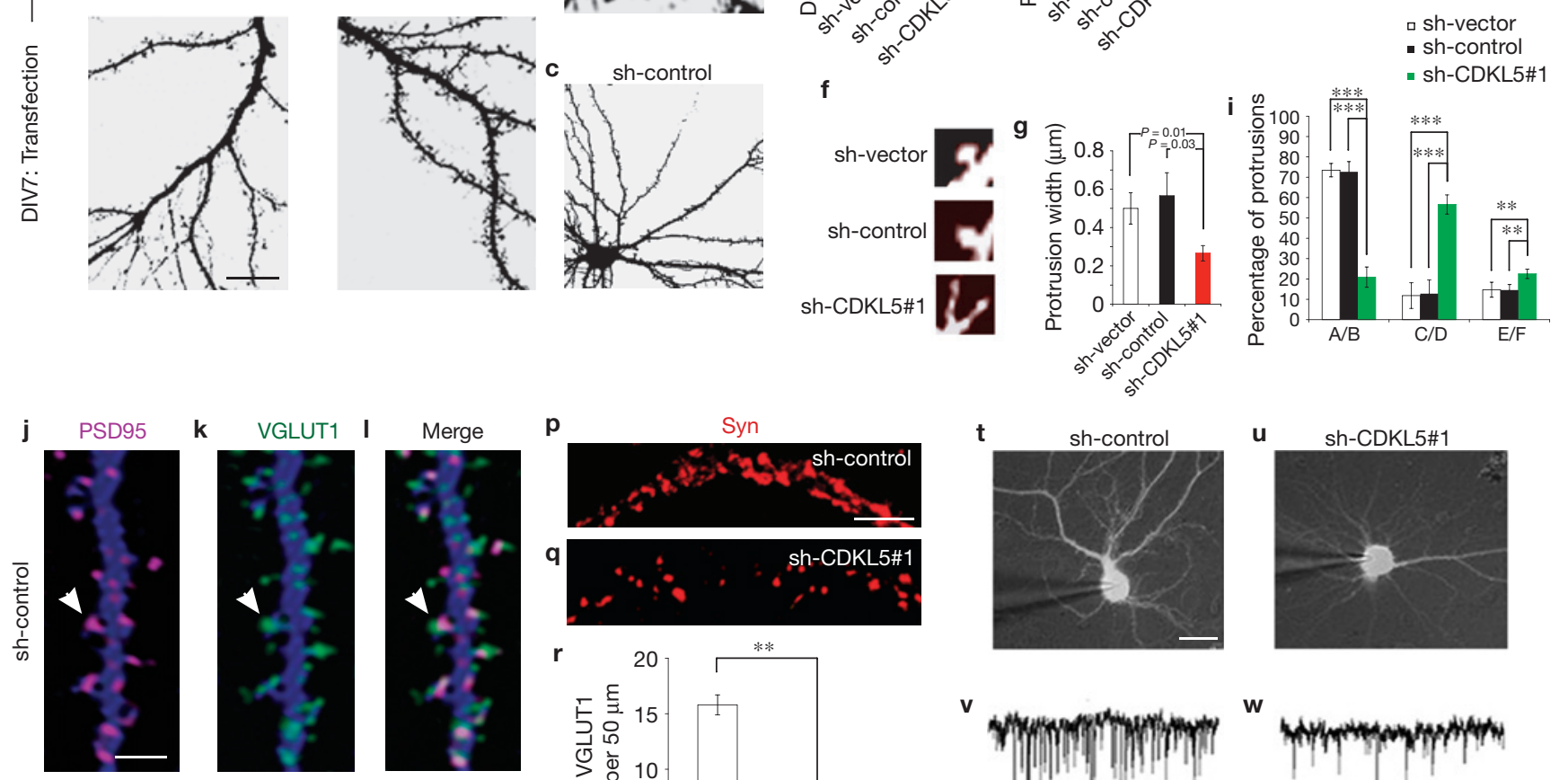

$\mathbf{t}$
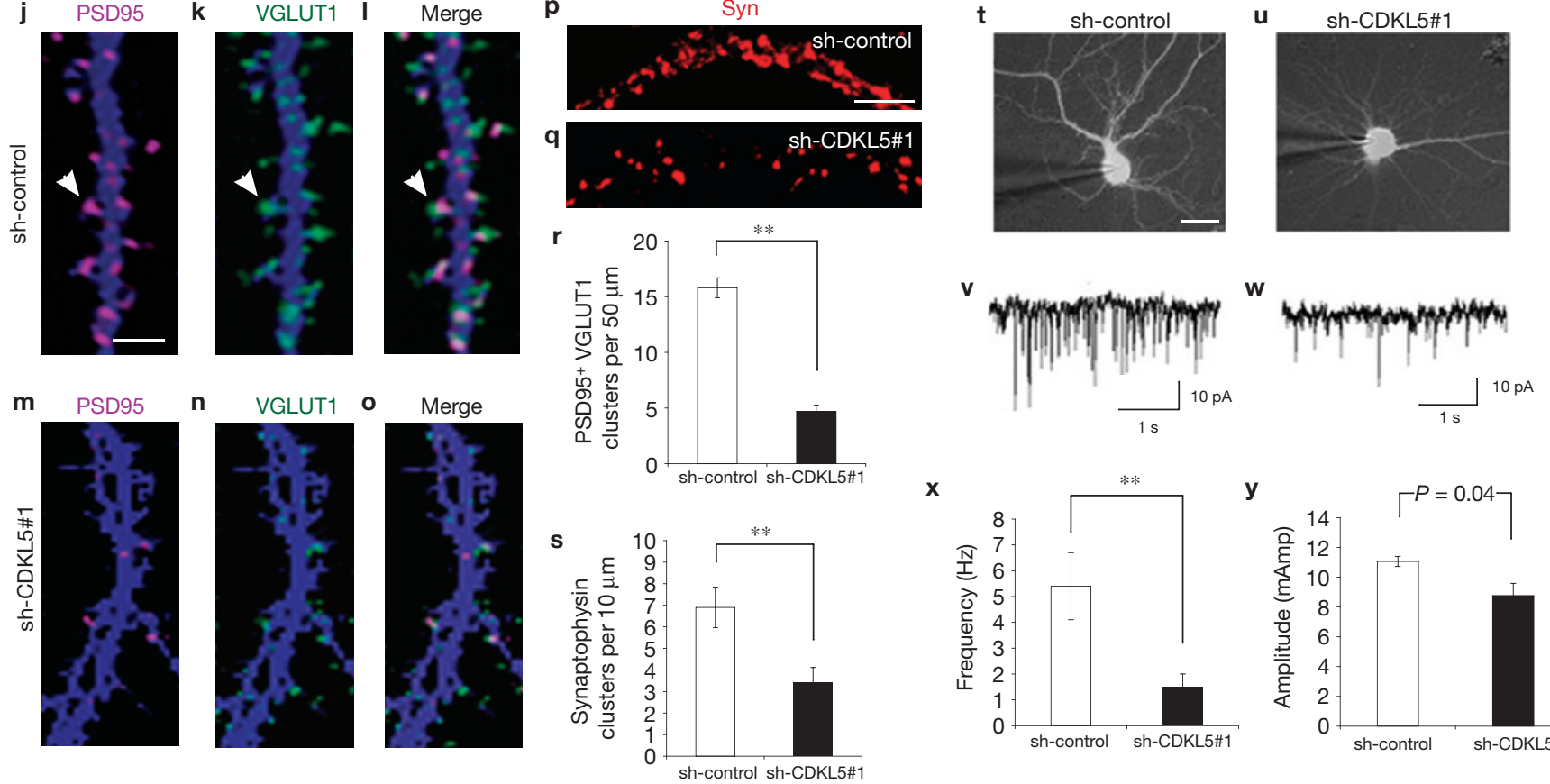

$\mathbf{X}$

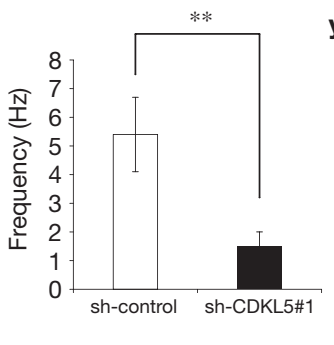

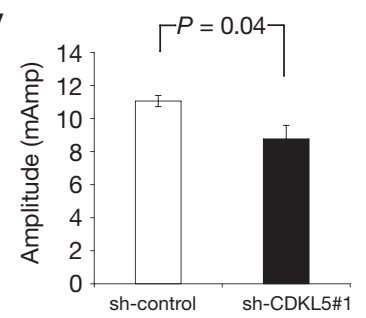

Figure 2 CDKL5 knockdown alters spine morphology and synaptic activity. (a-i) Effects of CDKL5 knockdown on spine morphology. (a-c) Representative images of primary hippocampal neurons transfected at DIV7 with sh-vector, sh-control or sh-CDKL5\#1 and labelled for GFP at DIV14. The bottom panels in $\mathbf{a}$ and $\mathbf{b}$ show a higher magnification of the panels above. The top right panels in $\mathbf{b}$ represent higher magnifications of $\mathbf{a}$ (top) and $\mathbf{b}$ (middle and bottom) and show the spine phenotype in single GFP-positive dendrites expressing either sh-vector or sh-CDKL5\#1. The arrowheads point to dendritic protrusions. (d,e) Quantification of dendritic protrusion density (d) and length (e) of sh-vector-, sh-control- and sh-CDKL5\#1-transfected neurons. The bars show mean \pm s.e.m. $n=15$ neurons for sh-vector and $n=18$ neurons for sh-control and sh-CDKL5\#1 (***P $P<0.001, t$-test). $n$ is derived from four independent experiments. (f,g) $C D K L 5$ downregulation decreases spine width. The bars in $\mathbf{g}$ show mean \pm s.e.m. $n=15$ neurons for sh-vector and $n=18$ neurons for sh-control and sh-CDKL5\#1. $n$ is derived from four independent experiments. (h) Schematic representation of dendritic protrusion morphology categories. (i) Quantification of the mean percentage of spines in each morphology category in neurons expressing sh-vector, sh-control or sh-CDKL5\#1. The bars show mean \pm s.e.m. $n=15$ neurons for sh-vector and $n=18$ neurons for sh-control and sh-CDKL5\#1 ${ }^{(* * *} P<0.001,{ }^{* *} P<0.01, t$-test). $n$ is derived from four independent experiments. (j-s) CDKL5 knockdown affects excitatory synapse number. Primary hippocampal neurons were transfected at DIV7 with sh-control or sh-CDKL5\#1 and immunostained at DIV14 with the indicated antibodies. I and $\mathrm{o}$ represent merged images. The arrowheads in $\mathbf{j}-1$ indicate co-localization of PSD95 with VGLUT1 at dendritic spines. The bars in $\mathbf{r}$ and $\mathbf{s}$ show mean \pm s.e.m. $n=16$ neurons for sh-control and sh-CDKL5\#1 ( ${ }^{* *} P<0.01, t$-test). $n$ is derived from three independent experiments. (t-y) CDKL5 knockdown in DIV15 cultured neurons reduced the frequency $(\mathbf{v}-\mathbf{x})$ and the amplitude $(\mathbf{v}, \mathbf{w}, \mathbf{y})$ of mEPSCs. $n=9$ neurons for sh-control and $n=13$ neurons for sh-CDKL5\#1 ( ${ }^{* *} P<0.01, t$-test). $n$ is derived from two independent experiments. Data are presented as means \pm s.e.m. Scale bars, $10 \mu \mathrm{m}$ (a (top and bottom), j, p, t); $5 \mu \mathrm{m}$ (b (top right)). 
a

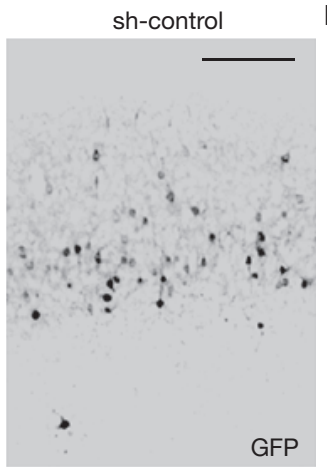

e

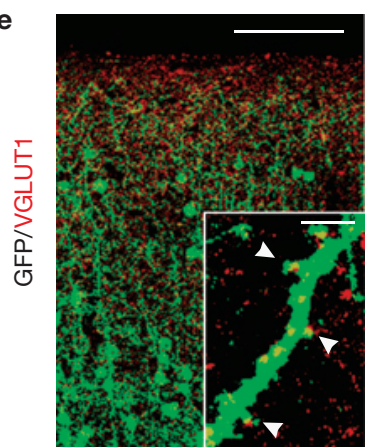

b
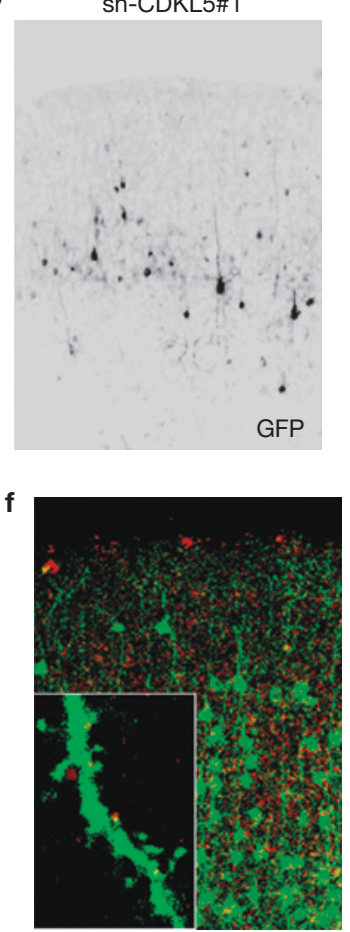

c

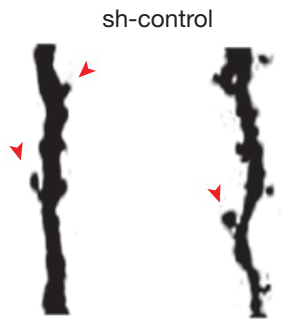

d

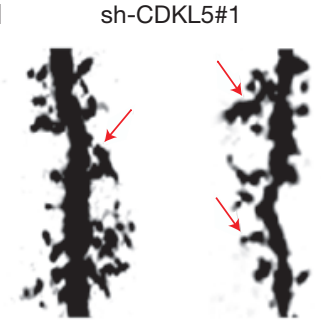

g

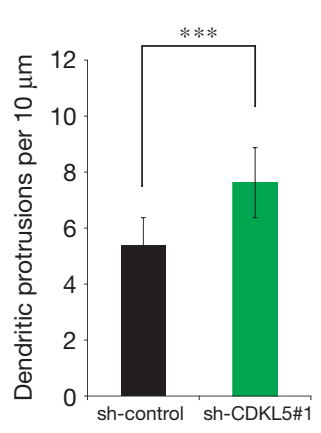

h

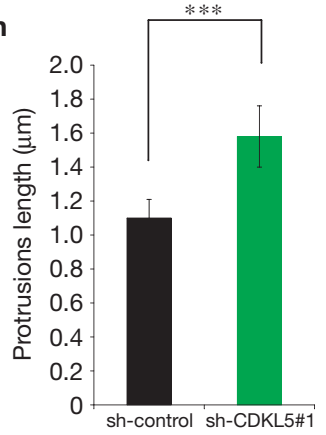

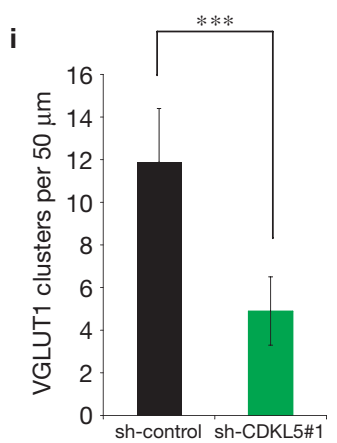

Figure 3 CDKL5 knockdown impairs spine structure and excitatory synapse density in vivo. $(\mathbf{a}, \mathbf{b})$ Representative confocal images of coronal slices of P11 mouse brains transfected with sh-control or sh-CDKL5\#1 by in utero electroporation at E13.5. Many transfected GFP-positive cells were detected in each transfection condition. (c,d) Representative images of single dendrites of GFP-positive cortex pyramidal neurons expressing sh-control or sh-CDKL5\#1. The arrows and arrowheads point to morphologically normal and aberrant spines, respectively. (e,f) Representative confocal images of cortex pyramidal neurons transfected with either sh-control or sh-CDKL5\#1 and immunostained

maturation $^{24,25}$, as a good starting point. Induction of NGL-netrin-G interaction promotes synapse formation ${ }^{26,27}$. In cells transfected with CDKL5-V5 and the intracellular domain of each of the NGLs, all 3 members were immunoprecipitated by the V5 antibody (Fig. 4a). In particular, CDKL5 binds specifically to a stretch of 5 amino acids located at the amino-terminal side of the intracellular domain (Fig. 4b-d). We also screened other PSD-enriched proteins that were directly or indirectly implicated in RTT-related disorders for CDKL5 interaction, but the results were negative (data not shown).

This prompted us to particularly characterize the association of CDKL5 with NGL-1. A co-clustering assay performed in cells co-transfected with GFP-CDKL5 and Myc-NGL-1 indicated that these proteins form intracellular and surface co-clusters (Fig. 4e-i). In fact, whereas CDKL5 localized in the cytoplasm when transfected alone, co-expression with NGL-1 resulted in CDKL5 translocation to the cell membrane and exact overlapping with NGL-1 (Fig. 4g,h). Further tests in a more physiological context showed that in hippocampal neurons both proteins co-localized precisely at dendritic spines (Fig. 4j). Similarly, Myc-NGL-1 also highly co-localized with endogenous CDKL5 (Fig. $4 \mathrm{k}-\mathrm{m}$ ). Next, the interaction of the proteins was assessed using P4 synaptosome brain extracts. Indeed, NGL-1 was coimmunoprecipitated by the CDKL5 antibody (Fig. 4n), indicating that for GFP and VGLUT1. The insets show a higher magnification of the main panels to a single neurite. The arrowheads in the inset of e show co-localization of CDKL5 and VGLUT1 at dendritic spines. (g,h) Quantitative analysis of dendritic protrusion density and length of GFP-positive cortex pyramidal neurons. The bars show mean \pm s.e.m. $n=15$ neurons for each condition ( ${ }^{* * *} P<0.001, t$-test). $n$ is derived from three independent experiments. (i) Quantitative analysis of VGLUT1 clusters in GFP-positive cortex pyramidal neurons. The bars show mean \pm s.e.m. $n=9$ neurons for each condition ( ${ }^{* * *} P<0.001, t$-test). $n$ is derived from three independent experiments. Scale bars, $100 \mu \mathrm{m}$.

NGL-1 assembles in the CDKL5 complex in vivo. Notably, both proteins even directly interact as ascertained by a pulldown assay (Fig. 4o).

These data prompted us to speculate that CDKL5 might phosphorylate NGL-1. At first, we analysed whether NGL-1 is phosphorylated in mouse brain by performing two-dimensional (2D) SDS-polyacrylamide gel electrophoresis (SDS-PAGE) from P10 extracts (Fig. 5a). NGL-1-specific staining was resolved in two protein spots. As the addition of the calf intestinal alkaline phosphatase (CIP) robustly reduced the intensity of only spot 2, whereas spot 1 increased its relative signal, we concluded that spot 2 represented the phosphorylated form of NGL-1 (Fig. 5a).

Subsequently, we subjected the entire or parts of the NGL-1 intracellular domain to Phos-tag staining. Interestingly, only the entire and the $\mathrm{C} 2 \mathrm{~N}$-terminal part produced an additional upper band that presents a putatively phosphorylated protein as confirmed by its loss after CIP treatment (Fig. 5b). These results indicate that NGL-1 is present with a mono-phosphorylated form both in vitro and in vivo.

\section{CDKL5 phosphorylates NGL-1 at Ser 631}

The C2 N-terminal domains of NGLs have several amino acids conserved between the family members, including Ser 600, Ser 620 and Ser 631. We mutated these serines into non-phosphorylable alanines 


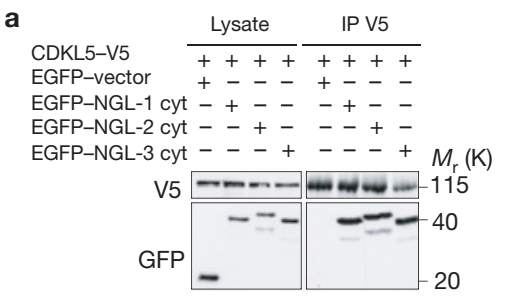

b

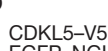

EGFP-NGL-1

EGFP-NGL-1 C2

EGFP-NGL-1 1555

EGFP-NGL-1 1570

EGFP-NGL-1 1 RTVEII

EGFP-NGL-1 ${ }^{\triangle T G D T P M}$

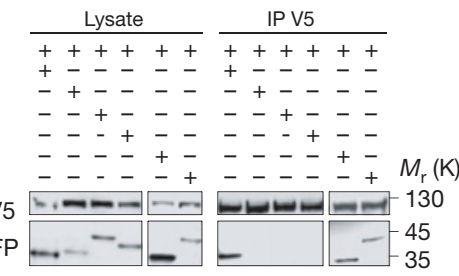

C

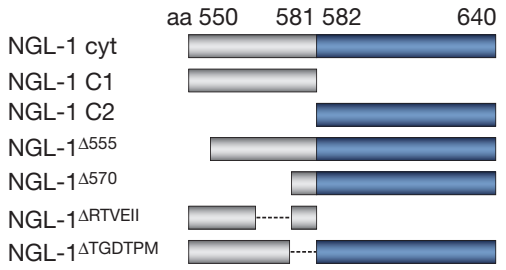

NGL-1 1555

NGL-1 1570

NGL-1 ${ }^{\triangle T G D T P M}$

DIV15 hippocampal neurons
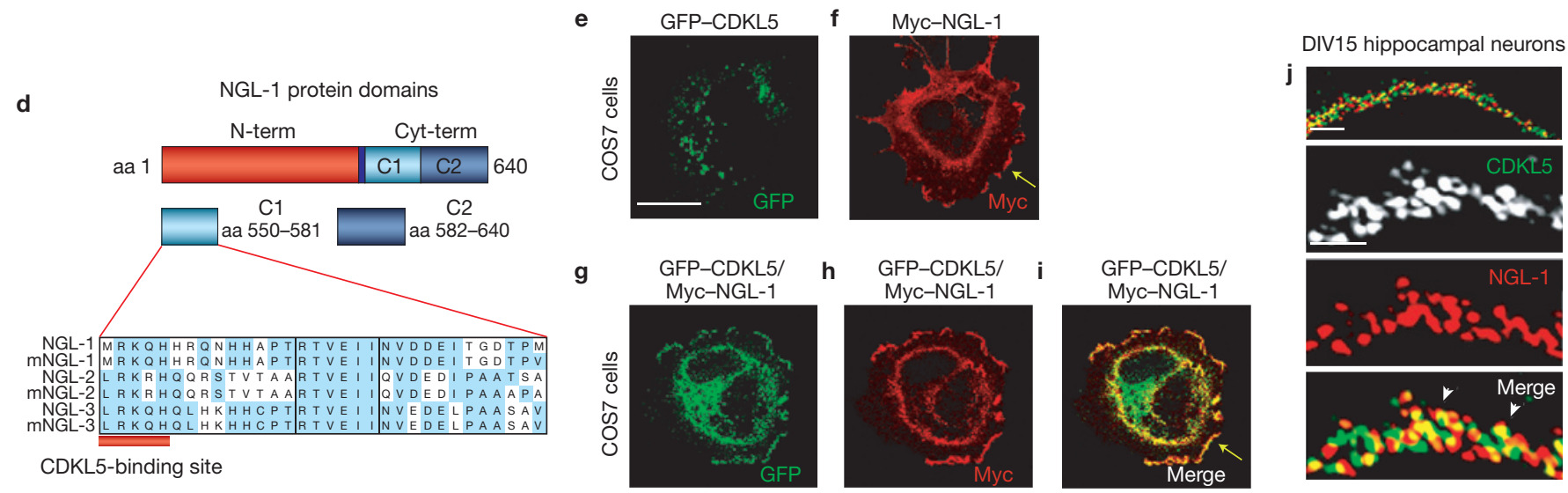

Mouse brain synaptosomes
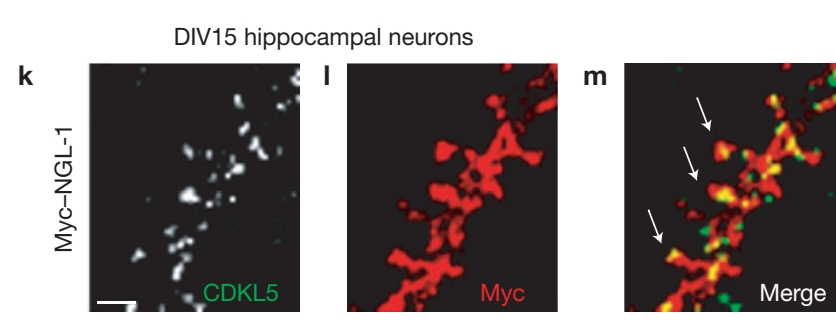

Figure 4 CDKL5 and NGL-1 interact in vitro and in vivo. (a) HEK293T cells were co-transfected with CDKL5-V5 and EGFP-NGL-1, -2 and -3 constructs representing their intracellular cytoplasmic region (cyt). Cell lysates were immunoprecipitated with anti-V5 antibody. Western blots were probed with anti-V5 or anti-GFP-HRP antibody. (b) HEK293T cells were co-transfected with CDKL5-V5 and various EGFP-NGL-1 cyt deletion constructs. Cell lysates were immunoprecipitated with anti-V 5 antibody. Western blots were probed with anti-V5 or anti-GFP-HRP antibody. (c) Schematic representation of deletion clones used for determining the CDKL5-binding site in the intracellular C-terminal domain of NGL-1. aa, amino acid. (d) Schematic representation of NGL-1 protein together with its intracellular C-terminal domain (Cyt-term) and CDKL5-binding site. (e-i) Co-clustering assay in COS-7 cells expressing GFP-CDKL5 (e), Myc-NGL-1 (f) and GFP-CDKL5 in combination with Myc-NGL-1 (g-i). (i) Merged image. The arrow shows redistribution of GFP-CDKL5 on the plasma membrane after co-transfection

and repeated the Phos-tag assays. Importantly, our results identified NGL-1 Ser 631, but not Ser 600 or Ser 620, to be phosphorylated (Fig. 5c). To obtain direct evidence of the CDKL5 ability to induce NGL-1 protein phosphorylation, the carboxy-terminal half of NGL-1 (amino acids 550-640) was purified and used for in vitro kinase assays. Interestingly, NGL-1 is readily phosphorylated in the presence of CDKL5. In contrast, the mutant S631A was not phosphorylated by CDKL5 (Fig. 5d). The catalytic activity of CDKL5 has been reported to be involved in its autophosphorylation in vitro ${ }^{16,18}$. Consistent with this, we observed autophosphorylation when CDKL5 was added (Fig. 5d).

To address whether the NGL-1 ${ }^{\mathrm{S} 631 \mathrm{~A}}$ mutant could be coimmunoprecipitated by CDKL5, we analysed their interaction. It
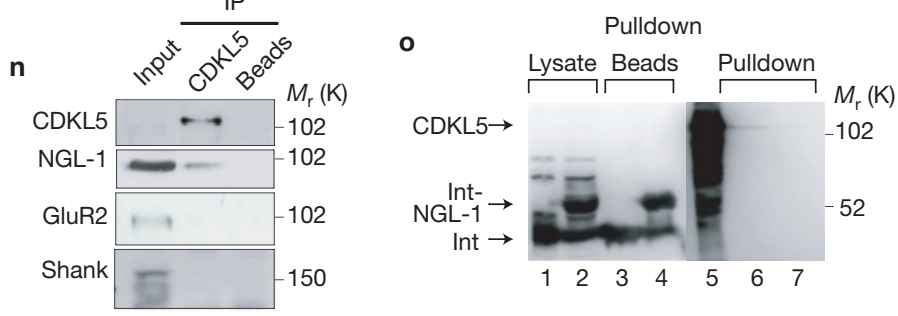

with NGL-1. (j) Primary hippocampal neurons labelled at DIV15 for CDKL5 and NGL-1. The arrowheads in the bottom image show co-localization of CDKL5 with NGL-1. (k-m) Primary hippocampal neurons transfected with Myc-NGL-1 at DIV7 and seven days later labelled for CDKL5 and Myc-NGL-1 to detect endogenous CDKL5 and recombinant Myc-NGL-1 protein. The arrows in $\mathbf{m}$ point to a region of co-localization between CDKL5 and NGL-1 at dendritic spines. (n) Interaction of endogenous NGL-1 with CDKL5 by co-immunoprecipitation from synaptosome lysates of P4 mouse brain. Anti-GluR2 and anti-Shank antibody stainings served as a negative control. (o) Intein fusion protein of NGL-1 (amino acids 550-640) pulls down CDKL5. Lanes 1 and 2, cellular lysate of IPTG-induced samples; lanes 3 and 4 , intein or intein-NGL-1-immobilized agarose beads; lane 5, CDKL5 input; lanes 6 and 7, CDKL5 pulldown induced by intein-NGL-1 or intein alone. Scale bars, $10 \mu \mathrm{m}$ (e); $5 \mu \mathrm{m}$ (j (for both bars), k). Uncropped images of blots are shown in Supplementary Fig. S8.

is noteworthy that GFP-CDKL5 failed to co-immunoprecipitate NGL- ${ }^{\text {S631A }}$, thus suggesting the relevance of the phoshorylation for reinforcing the interaction (Fig. 5e). To gain further support, we analysed the NGL-1-binding ability of various CDKL5 deletion mutants. Notably, none of the mutants lacking the kinase domain or its activity was able to immunoprecipitate NGL-1 (Supplementary Fig. S4), suggesting that the kinase domain and CDKL5 autophosphorylation are necessary for the stable interaction of CDKL5 with NGL-1.

We next investigated the phosphorylation level of NGL-1 in a fibroblast cell line derived from a female patient who carried an $\mathrm{X} ; 7$ chromosome translocation that truncated CDKL5 and lacked functional CDKL5 due to inactivation of the normal X chromosome 


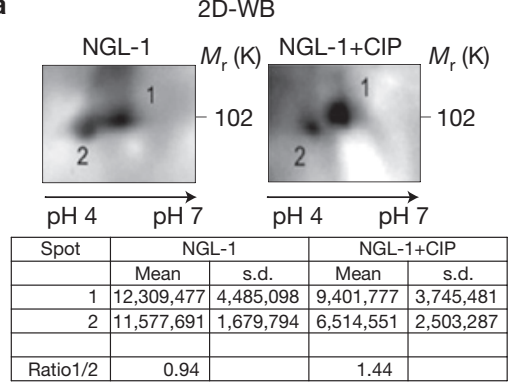

d

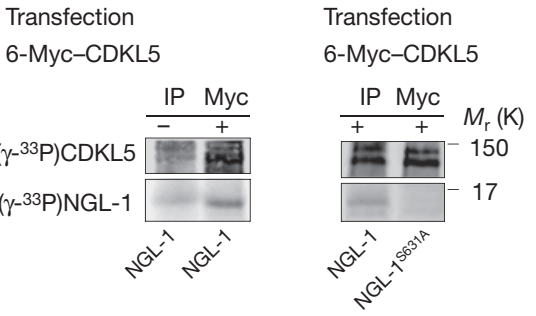

f

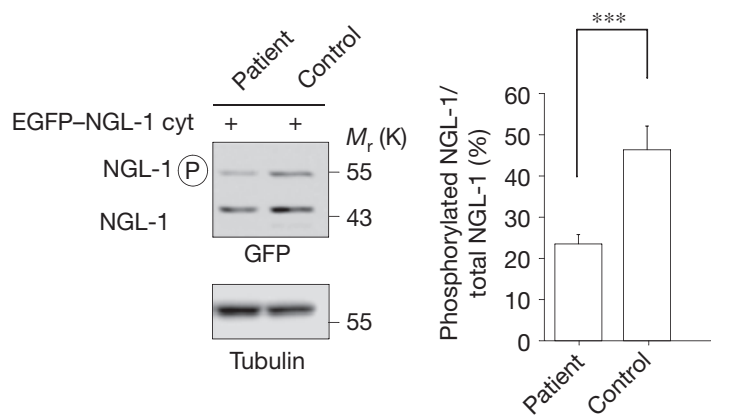

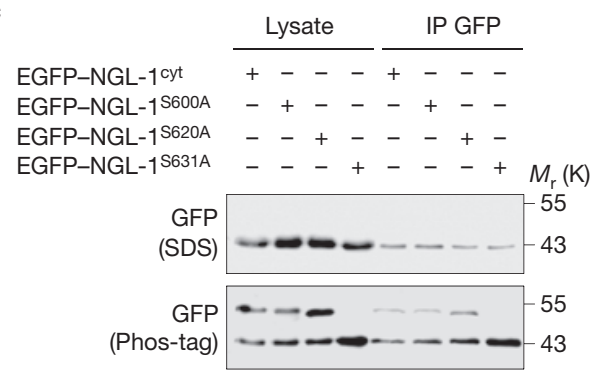

e
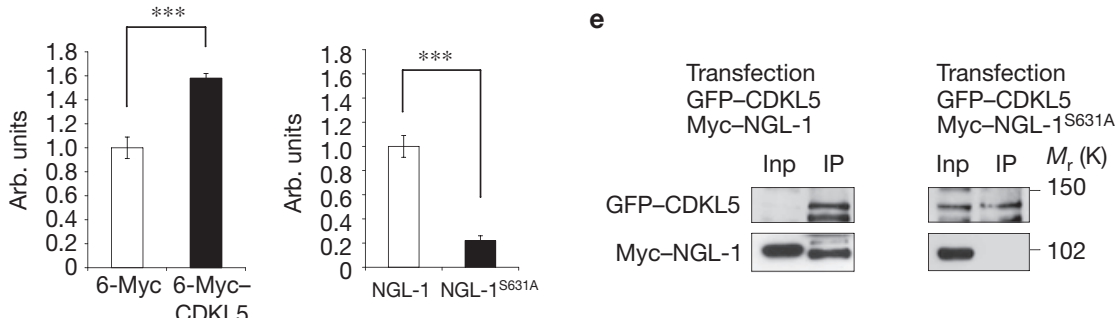

Figure 5 CDKL5 mediates phosphorylation in NGL-1 at Ser 631. (a) Total brain extracts were left untreated (left image) or were treated with CIP right image), subjected to $2 \mathrm{D}$ gel electrophoresis and analysed by immunoblotting with anti-NGL-1 antibody. The table reports the results of the densitometric scanning quantification of spots 1 and 2 and the ratio (1/2) of both, in control and CIP-treated samples. (b) Immunoprecipitation assays performed with anti-GFP antibody of HEK293T cells lysates transfected with the indicated plasmids and treated with CIP $(+)$ or left untreated $(-)$. Proteins were resolved in normal SDS-PAGE gels (upper panel) and in phosphate-affinity PAGE gels (lower panel). (c) HEK293T cells were transfected with EGFP-NGL-1 cyt, EGFP-NGL-1 ${ }^{\text {S600A }}$, EGFP-NGL-1 ${ }^{\text {S620A }}$ or EGFP-NGL-1 ${ }^{\text {S631A }}$ constructs. Immunoprecipitation assays were performed with anti-GFP antibody. Proteins were resolved in a normal SDS gel (upper panel) and a Phos-tag gel (lower panel). (d) In vitro kinase assay performed on HEK293T cell lysates transfected with the indicated plasmids and tested for NGL-1 (left blot) and NGL-1 ${ }^{\mathrm{S} 631 \mathrm{~A}}$ (right blot). The mean relative level \pm s.e.m.

(ref. 8). Importantly, the patient cells, when compared with normal control fibroblasts that express endogenous CDKL5, exhibited reduced levels of the NGL-1 phosphorylated form with respect to the total amount of the protein (Fig. 5f). Thus, these data suggest that the CDKL5-NGL-1 protein interaction is conserved in human cells and that NGL-1 is phosphorylated by CDKL5 also in fibroblasts. Along the same line, an increase of the NGL-1 phosphorylated form was detected when CDKL5 was overexpressed in the patient fibroblasts. This effect was not seen in normal control fibroblasts, suggesting that of NGL-1 and NGL-1 ${ }^{\mathrm{S} 631 \mathrm{~A}}$ phosphorylation is illustrated in a histogram ${ }^{* * *} P<0.001, t$-test). (e) Total cell lysates of HEK293T cells transfected with the indicated plasmids were subjected to immunoprecipitation assays with monoclonal anti-GFP and anti-Myc antibodies. (f) Control and CDKL5-negative patient fibroblast cells were transfected with EGFP-NGL-1 cyt. Total cell lysates were resolved in a Phos-tag SDS gel and probed with anti-GFP-HRP antibody. Anti-tubulin antibody staining served as a loading control. The relative NGL-1 phosphorylation level is presented as the mean \pm s.d. of four independent experiments compared with the total amount of NGL-1 ( ${ }^{* * *} P<0.001, t$-test). (g) Total cell lysates of both control and CDKL5-negative patient fibroblast cells were transfected with the indicated plasmids and resolved in a normal SDS gel or in a Phos-tag SDS gel. GFP protein levels were used to monitor transfection efficiency as indicated in NGL-1/GFP ratios (given in arbitrary units). The relative NGL-1 phosphorylation level is presented as the mean $\pm S D$ of three independent experiments compared with the total amount of NGL-1 (**P $P<0.01, t$-test) Uncropped images of blots are shown in Supplementary Fig. S8.

in these cells NGL-1 Ser 631 phosphorylation was already saturated by endogenous CDKL5 (Fig. 5g).

\section{NGL-1 binding to PSD95 is sustained by CDKL5 phosphorylation on Ser 631}

NGLs have a conserved C-terminal PDZ-binding domain (VQETQI), which specifically binds to PSD95 (ref. 27), a protein that plays a significant role in learning and memory ${ }^{29}$. NGL-1 spine-inducing capability is promoted by targeting PSD95 to new forming dendritic 
a
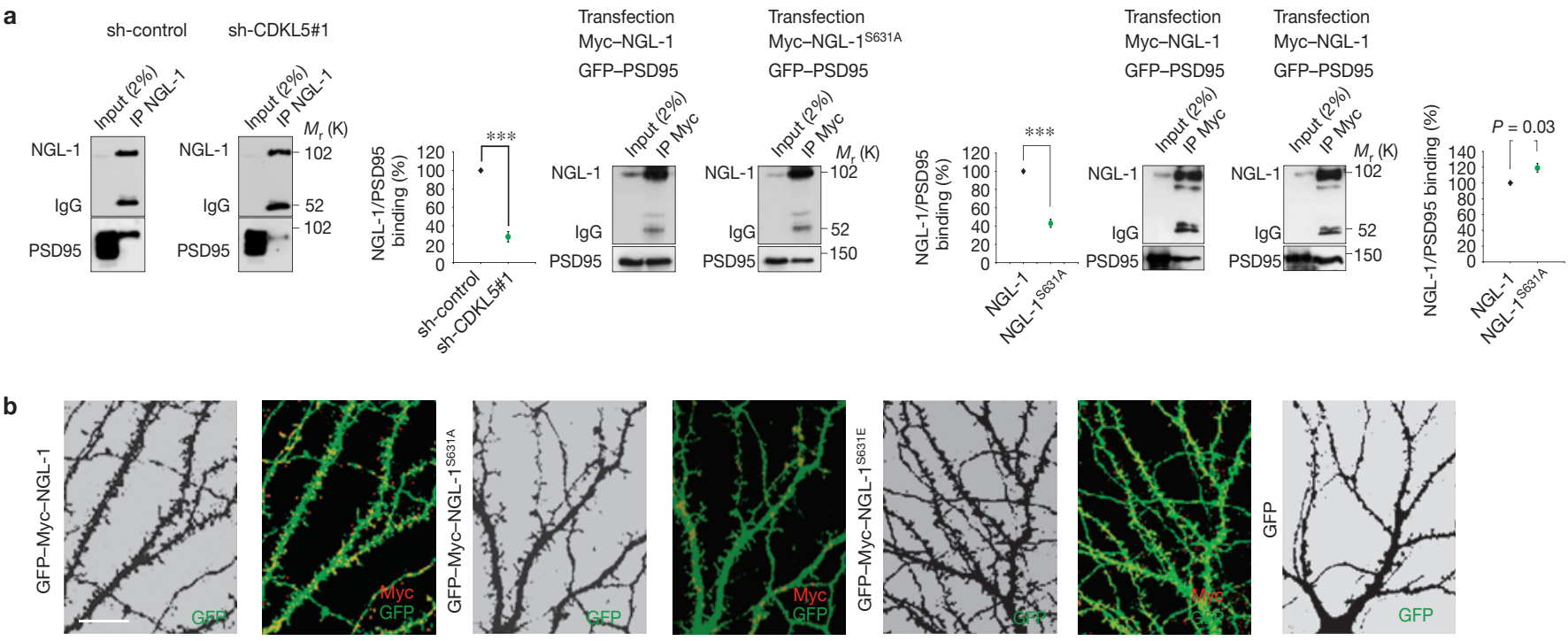

C
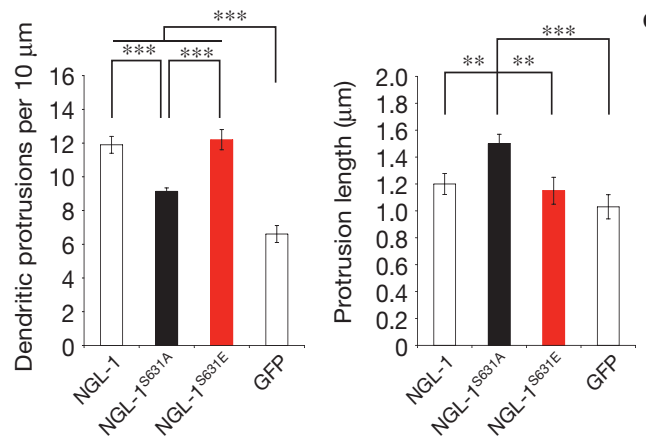

d
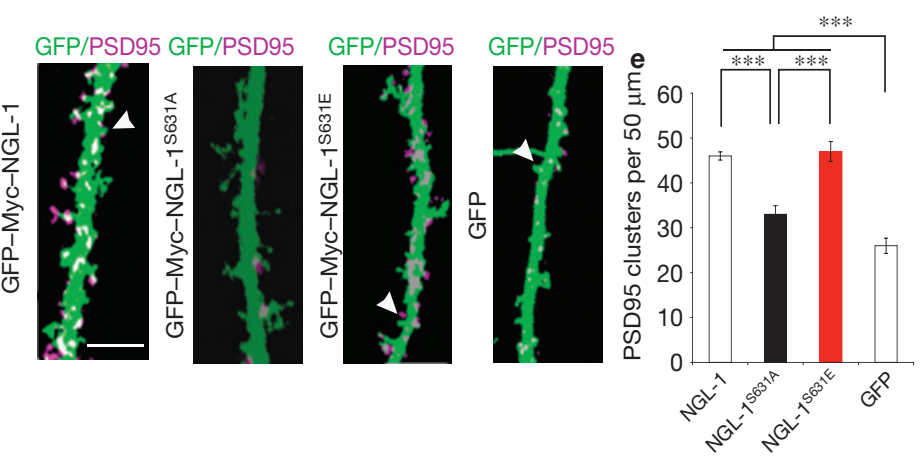

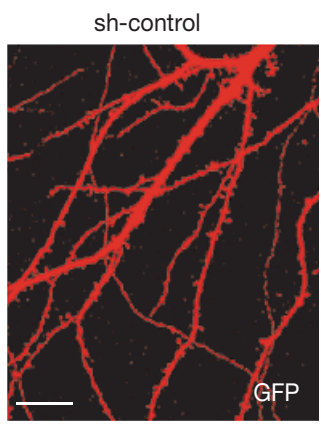

sh-CDKL5\#1

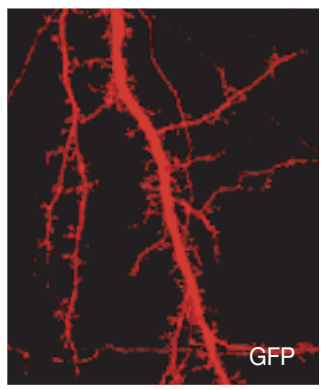

sh-CDKL5\#1+ GFP-NGL-1 1 S631E

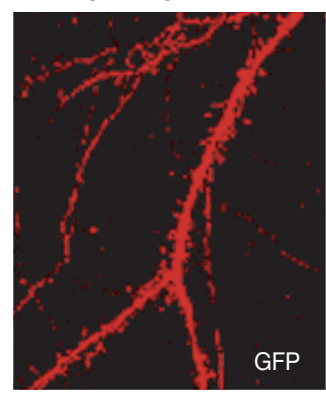

g

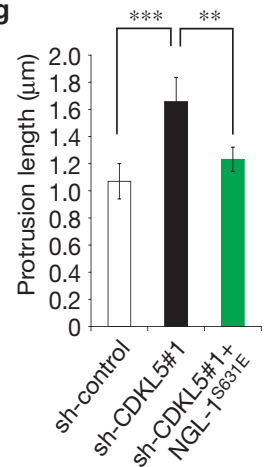

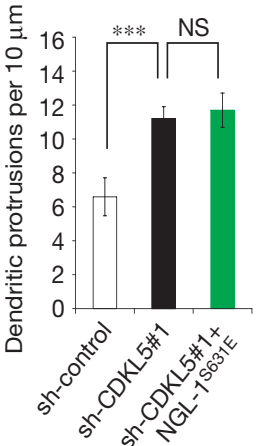

Figure 6 CDKL5-dependent phosphorylation of NGL-1 is necessary for NGL-1-PSD95 binding and correct spine morphogenesis. (a) CDKL5 knockdown reduces NGL-1-PSD95 binding in primary cortical neurons (left panels). Lysates of primary cortical neurons (DIV14) infected with either sh-control or sh-CDKL5\#1 were immunoprecipitated with antibodies against NGL-1 and immunoblotted with NGL-1 and PSD95 antibodies. CDKL5-dependent phosphorylation of NGL-1 on Ser 631 sustains NGL-1 binding to PSD95 (middle panels). Lysates of HEK293T cells double-transfected with GFP-PSD95 plus Myc-NGL-1 or Myc-NGL-1 ${ }^{\text {S631A }}$ were immunoprecipitated using an antibody against Myc and immunoblotted with Myc and PSD95 antibodies. Lysates of HEK293T cells double-transfected with GFP-PSD95 plus Myc-NGL-1 or Myc-NGL-1 ${ }^{\text {S631E }}$ were immunoprecipitated using an antibody against Myc and immunoblotted with Myc and PSD95 antibodies (right panels). Data are presented as means \pm s.e.m. ${ }^{* * *} P<0.001$. (b,c) Effect of overexpression of wild-type NGL-1, NGL-1 ${ }^{\mathrm{S} 631 \mathrm{~A}}$ and NGL-1 ${ }^{\mathrm{S} 631 \mathrm{E}}$ on dendritic protrusion density and morphology. Primary hippocampal neurons were transfected with the indicated plasmids at DIV7 and seven days later were stained for GFP and Myc. In c, the bars show mean \pm s.e.m. $n=12$ neurons for each ${ }^{* * *} P<0.001,{ }^{* *} P<0.01, t$-test). $n$ is derived from three independent experiments. (d,e) Primary hippocampal neurons were transfected with the indicated plasmids at DIV7 and were stained for GFP and PSD95 seven days after transfection. The arrowheads in $\mathbf{d}$ point to dendritic spines. The bars in e show mean \pm s.e.m. $n=10$ neurons for each $\left({ }^{* * *} P<0.001, t\right.$-test). $n$ is derived from three independent experiments. (f) NGL-1 ${ }^{\mathrm{S} 631 \mathrm{E}}$ partially rescues the phenotype of CDKL5 knockdown. Representative images of primary hippocampal neurons transfected at DIV7 with the indicated plasmids and labelled for GFP at DIV14. (g) Quantification of dendritic protrusion density and length of sh-control, sh-CDKL5\#1 and sh-CDKL5\#1 plus GFP-NGL-1 ${ }^{\text {S631E }}$-transfected neurons. The bars show mean \pm s.e.m. $n=12$ neurons for each ${ }^{* * *} P<0.001$, ${ }^{* *} P<0.01$; NS, not significant; $t$-test). $n$ derives from three independent experiments. Scale bars, $10 \mu \mathrm{m}$ (b,f); $5 \mu \mathrm{m}$ (d). Uncropped images of blots are shown in Supplementary Fig. S8. 
protrusions ${ }^{27}$. Given that CDKL5 interacts directly with NGL-1, we examined whether CDKL5 influences the interaction of these proteins. To do this, we performed a co-immunoprecipitation assay in cortical neurons infected at DIV7 with sh-CDKL5\#1. Seven days later, NGL-1 was immunoprecipitated and the amount of PSD95 in the complex was determined. Remarkably, we observed a robust reduction in the level of the NGL-1-PSD95 interaction in CDKL5-knockdown neurons (Fig. 6a). These results indicate that CDKL5 functions in promoting and/or maintaining the NGL-1-PSD95 protein association.

NGL-1 phosphorylated residue Ser 631 is very close to the terminal PDZ-binding domain and therefore located at a convenient site for regulating NGL-1-PSD95 interaction. To investigate this, we transfected cells with either NGL-1 or its S631A phosphomutant in combination with GFP-PSD95, immunoprecipitated the lysates for Myc-NGL-1 and measured the amount of associated PSD95. Indeed, the phospho-mutant showed a reduced level of association with PSD95 (Fig. 6a). Next, we examined the ability of the mutant to promote synaptogenesis in hippocampal neurons. Notably, dendritic protrusions were reduced in numbers and showed a significantly elongated morphology (Fig. 6b,c). These alterations are both qualitatively and quantitatively similar to the phenotype observed in CDKL5-downregulated neurons. Therefore, it is plausible that the NGL-1 ${ }^{\text {S631A }}$ mutant acts through a dominant-negative effect on endogenous NGL-1 and its ability to associate with PSD95.

To further strengthen this assumption, the phospho-mimetic NGL-1 mutant S631E was challenged in similar experiments. In particular, this mutant associated better with PSD95 although it was not able to significantly increase spine formation on overexpression (Fig. 6b,c). Despite that, when expressed in CDKL5-knockdown neurons, the mutant was able to restore nearly the correct length and morphology of dendritic spines, but not their numbers (Fig. 6f,g). Conversely, both phospho-mutants retained the ability to induce pre-synaptic terminals similar to the native NGL-1 when expressed by heterologous cells co-cultured with primary neurons ${ }^{27}$ (Supplementary Fig. S5). These findings are consistent with the NGL-1 extracellular domain being the main molecular player of this activity.

Overall, these data indicate that CDKL5 is critical for the maintenance of synaptic contacts, mainly by regulating the NGL-1 phosphorylated state and, thereby, its ability to bind PSD95 and stabilize their association.

\section{Patient-specific alterations in iPSC-derived neurons}

iPSC technology offers the possibility to model in vitro neurodevelopmental disorders by providing access to human neurons. Therefore, we set out to establish iPSC lines from two female patients diagnosed with atypical RTT caused by CDKL5 pathogenic mutations (R59X, L220P) and whose primary fibroblasts were already characterized ${ }^{20,30}$. Fibroblasts were infected with OCT4-, SOX2- and KLF4-expressing retroviruses and cultured in the presence of SR and FGF2. After 6-8 weeks, the iPSC-like clones expressed pluripotency markers and showed silencing of the retroviral exogenous genes (Fig. 7a and Supplementary Figs S6a,b and S7a,b). Pluripotent properties were also ascertained by testing the capability to differentiate into cells of the endoderm (SOX17-positive), mesoderm (SMA-positive) and neuroectoderm (TuJ1-positive) germ layers in vitro and the potential to generate teratomas (Supplementary Fig. S6c,d). Furthermore, correct chromosomal content was ascertained by karyotyping (Supplementary Fig. S7c).

Increasing evidence shows that female iPSCs may or may not retain the inactivated $\mathrm{X}$-chromosome status of the original fibroblasts ${ }^{31-33}$. Indeed, only iPSCs exclusively expressing the mutant CDKL5 allele will represent a coherent model of disease-affected cells. Thus, we investigated only those clones in which inherent X-chromosome inactivation was revealed by $\mathrm{H} 3 \mathrm{~K} 27 \mathrm{me} 3$ nuclear staining (Fig. 7a) and selected only cell lines that showed expression of either the mutant or the normal CDKL5 allele for further analysis. This approach allowed the generation of both pathogenic and healthy iPSCs from the same individual, providing stringent control cells for comparative analyses. A list of the cell lines used for each experiment is detailed in Supplementary Table S1.

Undifferentiated mutant and control iPSC clones did not show any evident difference (data not shown). We then induced their differentiation into neurons through the generation of neural-rosette intermediate neural progenitors and their subsequent differentiation for 55-60 days $^{34}$. In these conditions, neuronal cultures developed a highly intricate neuronal network highly enriched in forebrain-committed glutamatergic neurons (Fig. 7b-h). Interestingly, CDKL5 expression was found to strongly increase following neuronal differentiation (Fig. 8a). In particular, CDKL5 was found enriched in dendritic spines in well-matured neurons (Fig. 8b). These findings establish iPSC-derived neurons as an adequate in vitro model to study CDKL5-dependent biological mechanisms. Absence of CDKL5 did not impair the rate of neuronal differentiation or morphology (Fig. 8c-g). Interestingly, a significantly reduced number of synaptic contacts, indicated by the number of VGLUT1 and PSD95 puncta, was detected in the mutants (Fig. $8 \mathrm{~h}-\mathrm{m}$ ). To highlight spine morphology, neurons were infected with a lentivirus constitutively expressing GFP at low multiplicity. This staining permitted visualization of frequent abnormalities in the overall spine structure of mutant neurons (Fig. 8n-r). In particular, many of these altered spines did not present an evident pre-synaptic terminal (Fig. 8p,q). These results are well in line with those obtained for CDKL5-silenced mouse neurons and suggest a conserved role of this protein in neuronal structure homeostasis and activity.

\section{DISCUSSION}

In this study, we revealed how loss of CDKL5, the molecule responsible for the Hanefeld variant of RTT and early-onset intractable encephalopathy, impacts neuronal function. Our first observations indicate that CDKL5 mainly localizes to dendritic spines where it is juxtaposed to the PSD. Interestingly, CDKL5 was found in the human PSD (ref. 35; www.genes2cognition.org/HUMAN-PSD), suggesting that its localization at the synaptic PSD is evolutionarily conserved. Our data provide evidence that CDKL5 is necessary for correct genesis and maintenance of dendritic spines and for synapse formation. Indeed, knocking down CDKL5 significantly decreases the density and length of dendritic spines both in vitro and in vivo.

In line with the compromised development of spines, CDKL5downregulated neurons exhibit abnormal mEPSCs. Thus, our results reveal that CDKL5 is a key-limiting factor in regulating glutamatergic synapse formation and suggest that changes in excitatory synaptic strength might be responsible, at least in part, for the neurodevelopmental symptoms associated with its deficiency. 
a

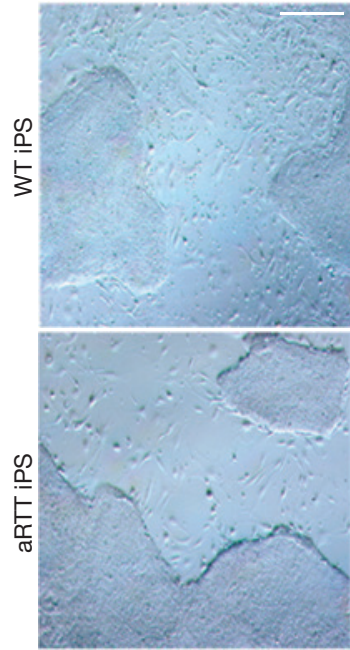

b
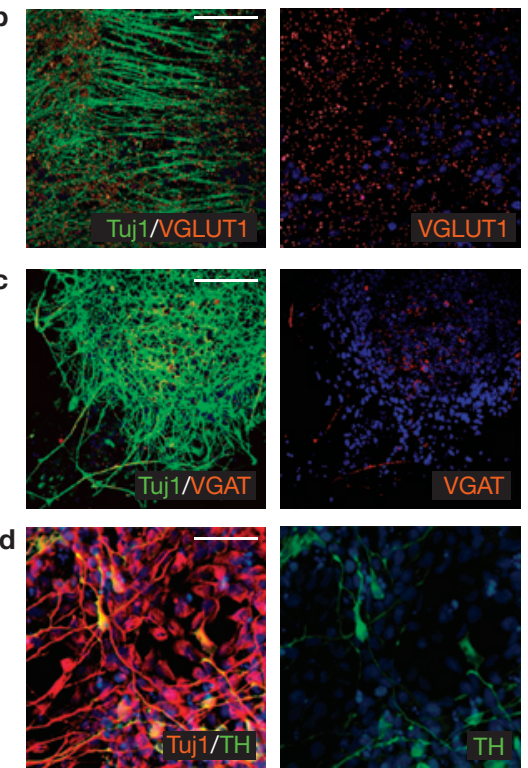

e

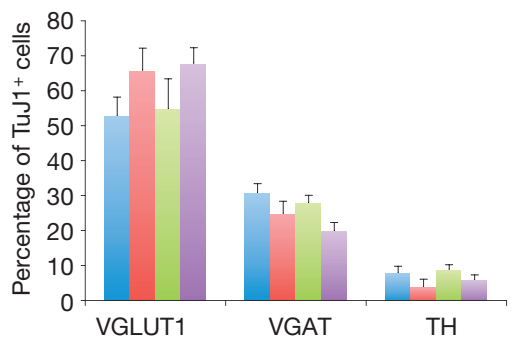

Figure 7 Assessment of pluripotency-associated markers in iPSCs and forebrain identity of iPSC-derived neurons. (a) Bright-field images of iPSC colonies in the growing phase. iPSCs express pluripotency markers such as OCT4, SOX2, NANOG and TRA-1-60 and exhibit a H3K27-positive inactivated X chromosome. (b-d) Cultured iPSC-derived neurons were immunolabelled with Tuj1 antibody in combination with either VGLUT1, VGAT or TH antibodies. The insets in the right panels of b and $\mathbf{c}$ show higher magnifications of a single neurite. (e) Quantification of the mean percentage of either VGLUT1, VGAT or TH Tuj1-positive cells for the neuronal progenies differentiated from four different iPSC

We identified CDKL5 as an interactor of NGLs, which are synaptic cell adhesion molecules (CAMs) that exert a pervasive role in synapse homeostasis ${ }^{24,27,36-38}$. Very intriguingly, we have previously found that
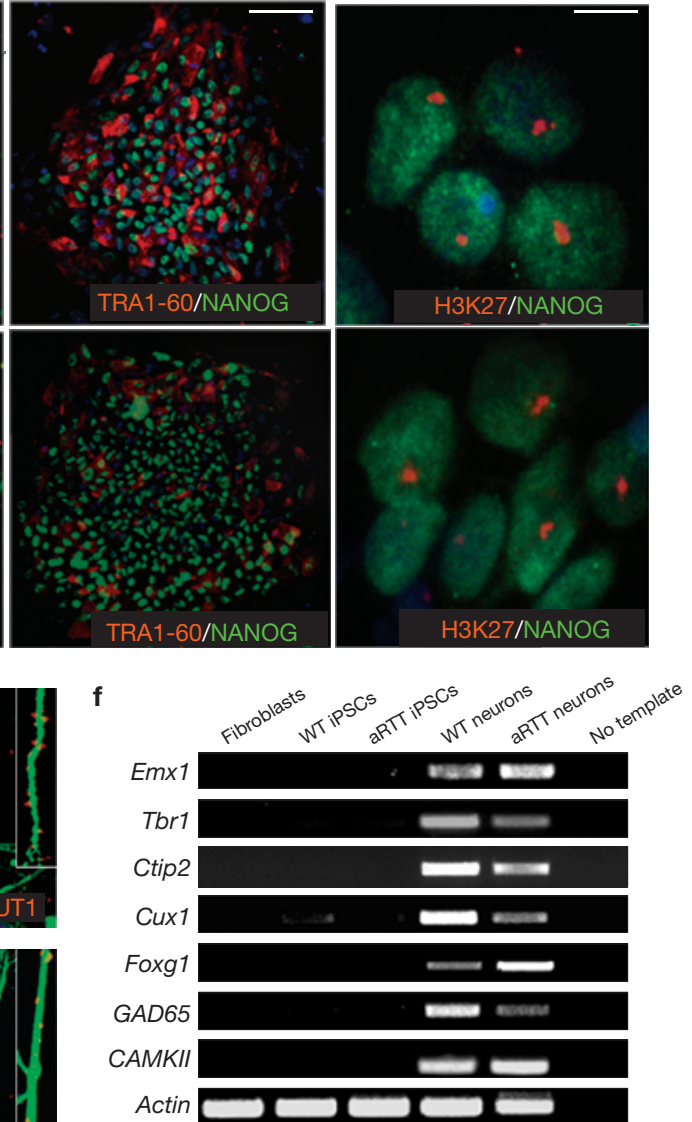

TuJ1/Ctip2

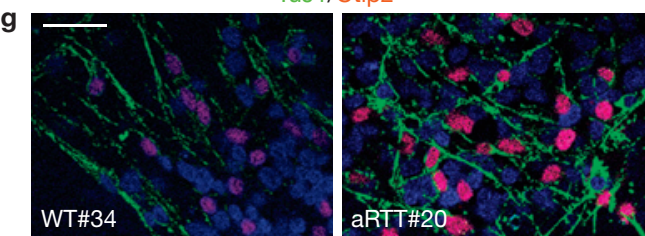

h

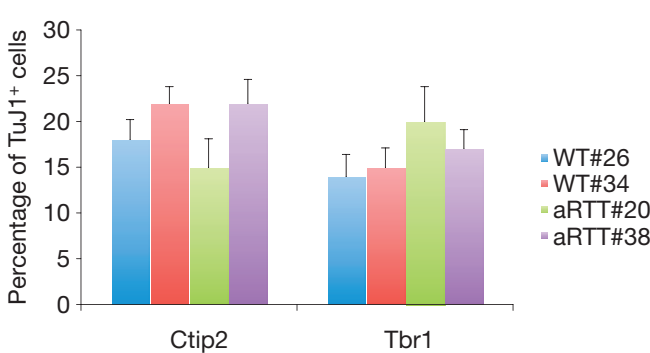

lines. The bars show mean \pm s.e.m. $n=390$ neurons ( $n$ is derived from three independent experiments per line). (f) RT-PCR expression analysis of specific brain area genes. iPSC-derived neurons expressed markers of forebrain identity as such Emx1, Tbr1, Ctip2, Cux1, CAMKII and Foxg1. (g) iPCS-derived TuJ1-positive neurons express the cortical layer-5 molecular marker Ctip2. (h) Quantification of the mean percentage of Ctip2 and Tbr1 expression in TuJ1-positive neurons. The bars show mean \pm s.e.m. $n=360$ neurons ( $n$ is derived from three independent experiments per line). Scale bars, $100 \mu \mathrm{m}$ (a, b,c,d); $50 \mu \mathrm{m}(\mathbf{d}, \mathbf{g})$; $10 \mu \mathrm{m}(\mathbf{a}, \mathbf{b}, \mathbf{c}, \mathbf{d})$.

NTNG1, which encodes the specific NGL-1 partner netrin-G1, was disrupted by a chromosome translocation in a patient with atypical RTT (ref. 23). It is then highly plausible that CDKL5 and NGL-1 


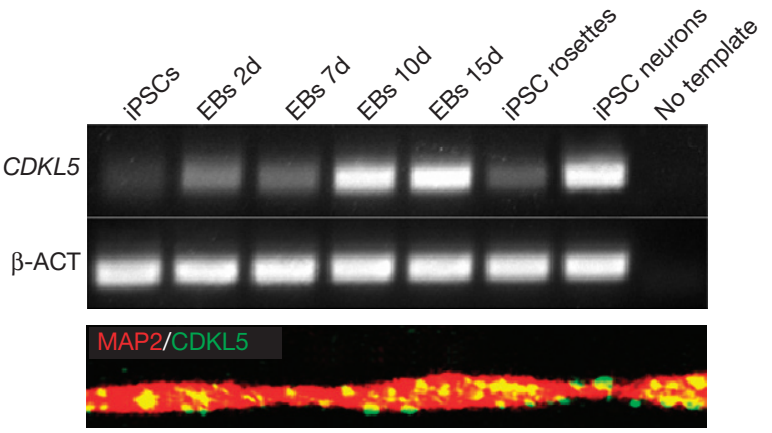

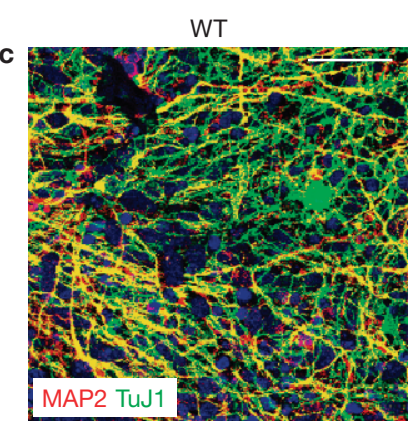

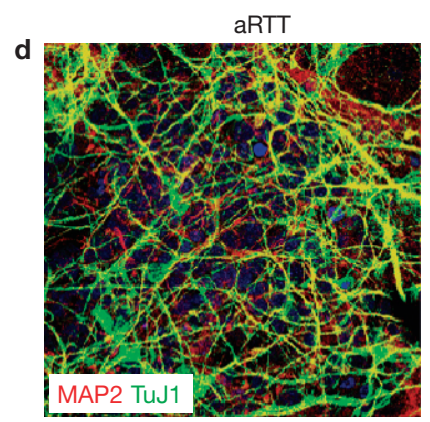

e

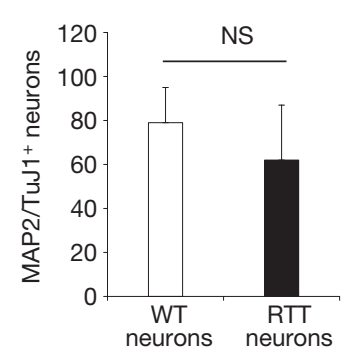

f

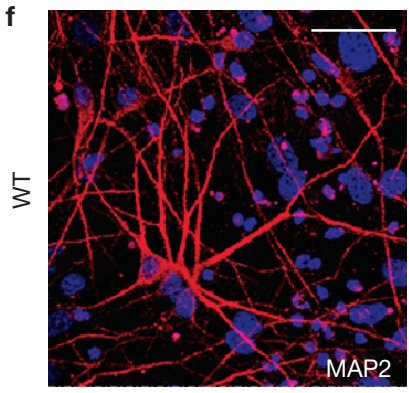

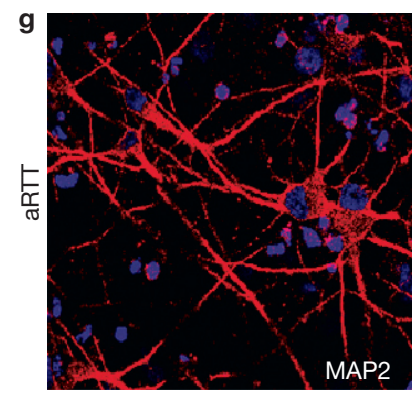

h

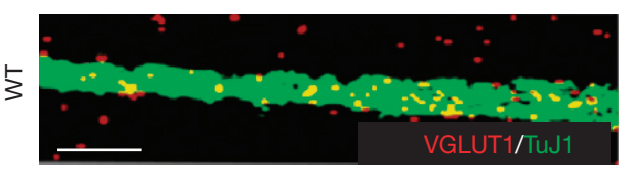

i

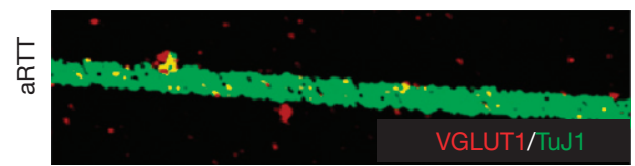

n

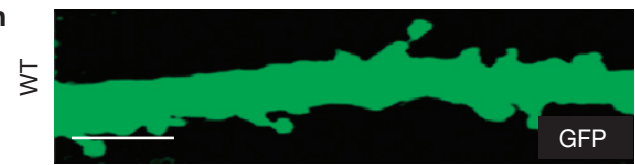

o

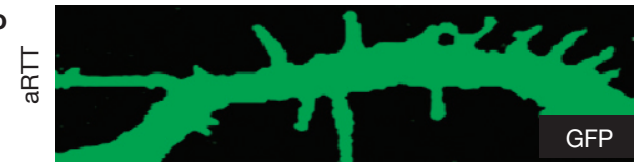

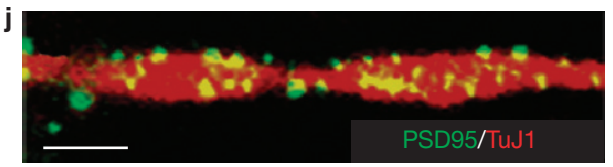
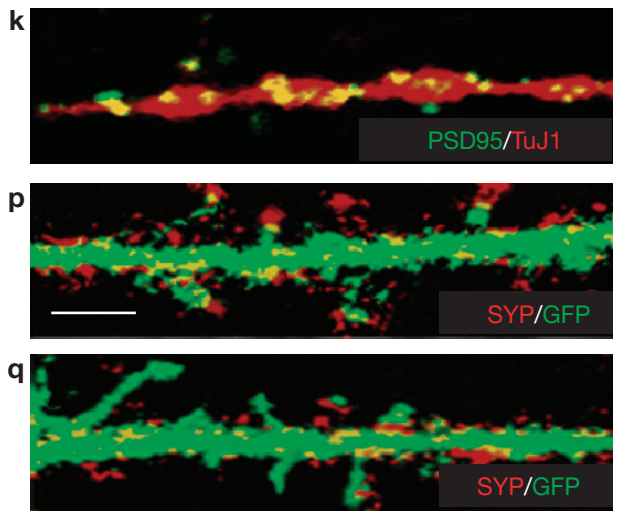

I

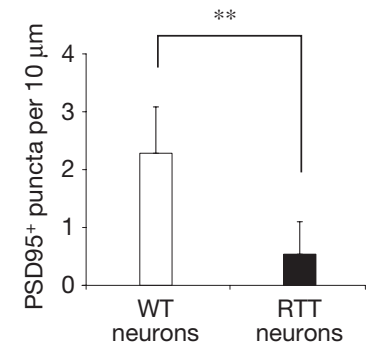

m

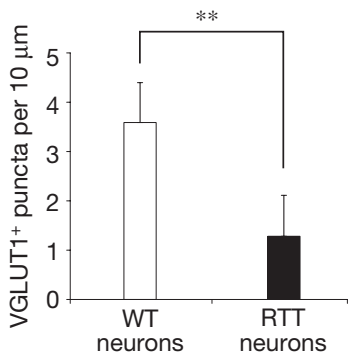

$r$

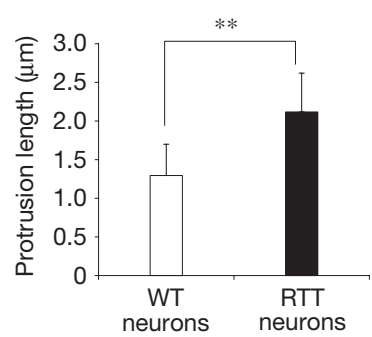

Figure 8 Patient-specific iPSC-derived neurons exhibit a normal differentiation rate, but aberrant spine structures. (a) The number of CDKL5 transcripts increased in embryoid bodies (EBs) and during neuronal differentiation. (b) CDKL5 immunofluorescence micrograph showing its localization enriched at the synapses of iPSC-derived neurons. (c,d) Low-magnification view of both affected and healthy iPSC-derived neuronal cultures immunodecorated for MAP2 and TuJ1. (e) The neuronal differentiation rate is not altered in aRTT. $n=250$ neurons ( $n$ is derived from five independent experiments per line); NS, not significant. $(\mathbf{f}, \mathbf{g})$ Representative images of 62-day-old wild-type and aRTT iPSC-derived neurons immunostained for MAP2. (h-k) High-magnification images on dendritic tracts of wild-type and aRTT iPSC-derived neurons processed

might work, at least in part, in a common pathway, thus providing a molecular framework explaining the similar neuropathological outcome of NTNG1 and CDKL5 mutations.

Our findings prompted us to search for pathogenic mutations in NGL-1 and -2 in patients with atypical RTT. No likely pathogenic changes were detected in these genes, suggesting that they are not a major contributor (data not shown). Although we did not detect NGL-1, for VGLUT1/TuJ1 and PSD95/TuJ1 antibody staining. (I,m) Quantitative analysis reveals a reduced number of both VGLUT1 $(\mathbf{h}, \mathbf{i})$ and PSD95 $(\mathbf{j}, \mathbf{k})$ puncta in the aRTT when compared with wild-type neurons. The bars show mean \pm s.e.m. $n=580$ spines. ( $n$ is derived from five independent experiments per line; ${ }^{* *} P<0.01, t$-test.) $(\mathbf{n}, \mathbf{0}) \mathrm{GFP}$ visualization permits the identification of aberrant spines with a very long and thin appearance in aRTT neurons. $(\mathbf{p}, \mathbf{q})$ Aberrant spines in aRTT neurons lack an evident presynaptic terminal as shown by immunofluorescence microscopy for synaptophysin (SYP). ( $r$ ) Quantification of the mean length of dendritic spines in wild-type and aRTT neurons. The bars show mean \pm s.e.m. $n=550$ spines ( $n$ is derived from five independent experiments per line; ${ }^{* *} P<0.01$, $t$-test). Scale bars, $50 \mu \mathrm{m}$ (c,f); $20 \mu \mathrm{m}$ (j); $10 \mu \mathrm{m}$ (h); $5 \mu \mathrm{m}$ (n, p).

-2 mutations, other groups reported deletions including NTNG1 in patients with intellectual disability and seizures ${ }^{39}$, NTNG1 missense mutations in autism ${ }^{40}$, and the association of NTNG1 non-coding single-nucleotide polymorphisms with schizophrenia ${ }^{41}$.

We provide evidence that CDKL5 phosphorylates NGL-1 on a unique serine, which is very close to the PDZ-binding domain that interacts with PSD95. This phosphorylation can also be extracted 
from a phospho-proteomic survey of mouse tissues ${ }^{28}$. So far, little evidence exists showing that the activity of synaptic CAMs is controlled by post-translational mechanisms. The discovery of a regulated phosphorylation process in NGL-1 opens the exciting possibility that the activity of other CAMs can be controlled by their phosphorylation state.

We demonstrated that CDKL5 is able to phosphorylate NGL-1 at Ser 631 both in vitro and in mouse brain. Importantly, the same results were obtained for human cells. In fact, CDKL5-negative patient fibroblasts exhibited reduced NGL-1 phosphorylation when compared with control cells. As NGL-1 phosphorylation was not completely absent, it is plausible to assume that other kinases substitute for CDKL5 in these cells. To overcome the limitations of using skin fibroblasts, we generated iPSC-derived neurons from two female patients with atypical RTT. Interestingly, in these neurons we detected a loss of synaptic contacts together with an increased number of aberrant dendritic spines, indicating that they represent a reliable system for studying CDKL5 function.

We showed that CDKL5-mediated phosphorylation of NGL-1 is necessary to ensure stable binding to PSD95. This mechanism resembles the phosphorylation dynamics of ion channels that mediate changes in the interaction with PSD95 (refs 42,43). Furthermore, phosphorylation of serine at position -9 outside the PDZ-binding domain in ephrin B ligands is required for the stable interaction with GRIP at the PSD and ensures correct synaptic activity ${ }^{44}$. These results mirror our findings and highlight a new aspect of fine-tuning protein-protein interaction by serine phosphorylation.

Interestingly, overexpression of a NGL-1 non-phosphorylable mutant leads to significant elongation of dendritic protrusions. This phenotype closely resembles the defects observed in CDKL5-silenced neurons, suggesting that the mutant could act in a dominant-negative manner leading to the disruption of the NGL-1-PSD95 interaction. Thus, these data indicate that synaptic strength and stability can be modified by altering the ratio between phosphorylated and unphosphorylated NGL-1 and, possibly, its homologues.

Our results are consistent with a model in which loss of CDKL5 elicits spine length and synaptic strength by regulating NGL-1-PSD95 interaction. On the other hand, CDKL5 interacts with Rac1 (ref. 21), which participates in stabilizing the actin cytoskeleton. Therefore, it is possible that Racl activity is altered by CDKL5 deletion. This is particularly relevant given that actin filament dynamics within the spines control their morphology and stability ${ }^{45}$. Thus, we cannot exclude that some aspects of the alterations described in this study can be ascribed to defects in such mechanisms. In agreement with this assumption, rescue of CDKL5-dependent spine deficits by a phospho-mimetic NGL-1 mutant is incomplete, suggesting that other mechanisms are dysregulated by CDKL 5 impairment.

We demonstrate that loss of CDKL5 severely affects spine morphology and reduces functional synaptic contacts, impairing neuronal activity.

We propose a model in which CDKL5-dependent phosphorylation on Ser 631 controls the association of NGL-1 with the postsynaptic molecular hub PSD95. In light of these findings, we provide mechanistic insights into how CDKL5 mutations can impact on neuronal function in atypical forms of RTT and other CDKL5-related neurodevelopmental disorders.

\section{METHODS}

Methods and any associated references are available in the online version of the paper.

Note: Supplementary Information is available in the online version of the paper

\section{ACKNOWLEDGEMENTS}

We gratefully acknowledge T. Bienvenu for providing primary fibroblasts from CDKL5 patients, H. Van Esch and H. Archer for providing DNA samples from patients, S. Freier, A. Walther, A. Grimme, U. Fischer and B. Moser for their excellent technical assistance, and L. Musante for helpful discussions. L. Pecciarini is acknowledged for karyotype analysis of the iPSC lines. This study was supported by the Telethon Foundation, Ministry of Health, IIT-SEED project, EuroRETT network to V.B. and the EU-FP7 project GENCODYS (241995) to V.M.K.

\section{AUTHOR CONTRIBUTIONS}

V.M.K. and N.R. initiated this study. S.R., M.H., N.R, V.M.K. and V.B. contributed to its conceptualization and V.M.K and V.B. directed it throughout; S.R. performed and analysed neuronal experiments. F.U. generated iPSCs and characterized iPSCderived neurons. M.H. performed and analysed immunoprecipitation assays and Phos-tag assays. M.H. and N.R. performed mutation search. G.S. was involved in mutagenesis experiments. D.B. performed electrophysiological recordings. A.S. performed electroporation experiments. C.M. and A.B. performed 2D gel analysis. E.G. produced the CDKL5-specific antibody. C.V. and C.S. assisted with the preparation of primary neurons. C.K-N. assisted with the in vitro phosphorylation assays. V.M.K., V.B., S.R., M.H. and N.R. interpreted the results. V.B., V.M.K. and S.R. wrote the manuscript.

\section{COMPETING FINANCIAL INTERESTS}

The authors declare no competing financial interests.

Published online at www.nature.com/doifinder/10.1038/ncb2566

Reprints and permissions information is available online at www.nature.com/reprints

1. Bienvenu, T. \& Chelly, J. Molecular genetics of Rett syndrome: when DNA methylation goes unrecognized. Nat. Rev. Genet. 7, 415-426 (2006).

2. Chahrour, M. \& Zoghbi, H. Y. The story of Rett syndrome: from clinic to neurobiology. Neuron 56, 422-437 (2007).

3. Amir, R. E. et al. Rett syndrome is caused by mutations in X-linked MECP2, encoding methyl-CpG-binding protein 2. Nat. Genet. 23, 185-188 (1999).

4. Francke, U. Mechanisms of disease: neurogenetics of MeCP2 deficiency. Nat. Clin. Pract. Neurol. 2, 212-221 (2006).

5. Tao, J. et al. Mutations in the X-linked cyclin-dependent kinase-like 5 (CDKL5/STK9) gene are associated with severe neurodevelopmental retardation. Am. J. Hum. Genet. 75, 1149-1154 (2004).

6. Weaving, L. S. et al. Mutations of CDKL5 cause a severe neurodevelopmental disorder with infantile spasms and mental retardation. Am. J. Hum. Genet. 75, 1079-1093 (2004).

7. Archer, H. L. et al. CDKL5 mutations cause infantile spasms, early onset seizures, and severe mental retardation in female patients. J. Med. Genet. 43, 729-734 (2006).

8. Kalscheuer, V. M. et al. Disruption of the serine/threonine kinase 9 gene causes severe X-linked infantile spasms and mental retardation. Am. J. Hum. Genet. 72, 1401-1411 (2003).

9. Córdova-Fletes, C. et al. CDKL5 truncation due to a $t(X ; 2)(p 22.1 ; p 25.3)$ in a girl with X-linked infantile spasm syndrome. Clin. Genet. 77, 92-96 (2010).

10. Scala, E. et al. CDKL5/STK9 is mutated in Rett syndrome variant with infantile spasms. J. Med. Genet. 42, 103-107 (2005).

11. Rademacher, $N$. et al. Identification of a novel CDKL5 exon and pathogenic mutations in patients with severe mental retardation, early-onset seizures and Rett-like features. Neurogenetics 12, 165-167 (2011).

12. Bahi-Buisson, N. et al. Key clinical features to identify girls with CDKL5 mutations. Brain 131, 2647-2661 (2008).

13. Van Esch, H., Jansen, A., Bauters, M., Froyen, G. \& Fryns, J. P. Encephalopathy and bilateral cataract in a boy with an interstitial deletion of Xp22 comprising the CDKL5 and NHS genes. Am. J. Med. Genet. A 143, 364-369 (2007).

14. Mei, D. et al. Xp22.3 genomic deletions involving the CDKL5 gene in girls with early onset epileptic encephalopathy. Epilepsia 51, 647-654 (2010).

15. Liang, J. S. et al. CDKL5 alterations lead to early epileptic encephalopathy in both genders. Epilepsia 52, 1835-1842 (2011).

16. Bertani, I. et al. Functional consequences of mutations in CDKL5, an X-linked gene involved in infantile spasms and mental retardation. J. Biol. Chem. 281, 32048-32056 (2008).

17. Montini, E. et al. Identification and characterization of a novel serine-threonine kinase gene from the Xp22 region. Genomics 51, 427-433 (1988). 
18. Mari, F. et al. CDKL5 belongs to the same molecular pathway of MeCP2 and it is responsible for the early-onset seizure variant of Rett syndrome. Hum. Mol. Genet. 14, 1935-1946 (2005).

19. Rusconi, L. et al. CDKL5 expression is modulated during neuronal development and its subcellular distribution is tightly regulated by the C-terminal tail. J. Biol. Chem 283, 30101-30111 (2008).

20. Ricciardi, S., Kilstrup-Nielsen, C., Bienvenu, T., Jacquette, A., Landsberger, N. \& Broccoli, V. CDKL5 influences RNA splicing activity by its association to the nuclear speckle molecular machinery. Hum. Mol. Genet. 18, 4590-4602 (2009).

21. Chen, Q. et al. CDKL5, a protein associated with Rett syndrome, regulates neuronal morphogenesis via Rac1 signaling. J. Neurosci. 30, 12777-12786 (2010).

22. Rusconi, L., Kilstrup-Nielsen, C. \& Landsberger, N. Extrasynaptic N-methylD-aspartate (NMDA) receptor stimulation induces cytoplasmic translocation of the CDKL5 kinase and its proteasomal degradation. J. Biol. Chem. 286, 36550-36558 (2011).

23. Borg, I. et al. Disruption of Netrin G1 by a balanced chromosome translocation in a girl with Rett syndrome. Euro. J. Human Genet. 13, 921-927 (2005).

24. Woo, J., Kwon, S. K. \& Kim, E. The NGL family of leucine-rich repeat-containing synaptic adhesion molecules. Mol. Cell Neurosci. 42, 1-10 (2009).

25. Kim, E. \& Sheng, M. The postsynaptic density. Curr. Biol. 19, R723-R724 (2009).

26. Lin, J. C., Ho, W. H., Gurney, A. \& Rosenthal, A. The netrin-G1 ligand NGL-1 promotes the outgrowth of thalamocortical axons. Nat. Neurosci. 6, 1270-1276 (2003).

27. Kim, S. et al. NGL family PSD-95-interacting adhesion molecules regulate excitatory synapse formation. Nat. Neurosci. 9, 1294-1301 (2006).

28. Huttlin, E. L. et al. A tissue-specific atlas of mouse protein phosphorylation and expression. Cell 143, 1174-1189 (2010).

29. Migaud, M. et al. Enhanced long-term potentiation and impaired learning in mice with mutant postsynaptic density-95 protein. Nature 396, 433-439 (1998).

30. Rosas-Vargas, H. et al. Impairment of CDKL5 nuclear localisation as a cause for severe infantile encephalopathy. J. Med. Genet. 45, 172-178 (2008).

31. Kim, K. Y., Hysolli, E. \& Park, I. H. Neuronal maturation defect in induced pluripotent stem cells from patients with Rett syndrome. Proc. Natl Acad. Sci. USA 108, 14169-14174 (2011).
32. Marchetto, M. C. et al. A model for neural development and treatment of Rett syndrome using human induced pluripotent stem cells. Cell 143, 527-539 (2010).

33. Tchieu, J. et al. Female human iPSCs retain an inactive $\mathrm{X}$ chromosome. Cell Stem. Cell 7, 329-342 (2010).

34. Colleoni, S. et al. Long-term culture and differentiation of CNS precursors derived from anterior human neural rosettes following exposure to ventralizing factors. Exp. Cell Res. 316, 1148-1158 (2010).

35. Bayés, A. et al. Characterization of the proteome, diseases and evolution of the human postsynaptic density. Nat. Neurosci. 14, 19-21 (2011).

36. Hering, H. \& Sheng, M. Dendritic spines: structure, dynamics and regulation. Nat. Rev. Neurosci. 2, 880-888 (2001).

37. Woo, J. et al. Trans-synaptic adhesion between NGL-3 and LAR regulates the formation of excitatory synapses. Nat. Neurosci. 12, 428-437 (2009).

38. Han, K. \& Kim, E. Synaptic adhesion molecules and PSD-95. Prog. Neurobiol. 84, 263-283 (2008).

39. Van Kuilenburg, A. B. et al. Analysis of severely affected patients with dihydropyrimidine dehydrogenase deficiency reveals large intragenic rearrangements of DPYD and a de novo interstitial deletion del(1)(p13.3p21.3). Hum. Genet. 125, 581-90 (2009)

40. O'Roak, B. J. et al. Sporadic autism exomes reveal a highly interconnected protein network of de novo mutations. Nature 485, 246-250 (2012).

41. Ohtsuki, T. et al. Association of polymorphisms in the haplotype block spanning the alternatively spliced exons of the NTNG1 gene at $1 \mathrm{p} 13.3$ with schizophrenia in Japanese populations. Neurosci. Lett. 435, 194-197 (2008).

42. Cohen, N. A., Brenman, J. E., Snyder, S. H. \& Bredt, D. S. Binding of the inward rectifier $\mathrm{K}+$ channel Kir 2.3 to PSD-95 is regulated by protein kinase A phosphorylation. Neuron 17, 759-767 (1996).

43. Chung, H. J., Huang, Y. H., Lau, L. F. \& Huganir, R. L. Regulation of the NMDA receptor complex and trafficking by activity-dependent phosphorylation of the NR2B subunit PDZ ligand. J. Neurosci. 24, 10248-10259 (2004).

44. Essmann, C. L. et al. Serine phosphorylation of ephrinB2 regulates trafficking of synaptic AMPA receptors. Nat. Neurosci. 11, 1035-1043 (2008).

45. Kennedy, M. B., Beale, H. C., Carlisle, H. J. \& Washburn, L. R. Integration of of biochemical signalling in spines. Nat. Rev. Neurosci. 6, 423-434 (2005). 


\section{METHODS}

Plasmids and constructs. pEGFP-hCDKL5, pEGFP-hCDKL5 ${ }^{\Delta \mathrm{N}}$, pEGFPhCDKL5 ${ }^{\Delta 525}$, pEGFP-hCDKL5 $5^{\Delta 781}, \quad$ pEGFP-hCDKL5 ${ }^{\Delta 832}$, pEGFP-hCDKL5 832-1030 and pEGFP-hCDKL5 ${ }^{\mathrm{K} 42 \mathrm{R}}$ were described previously ${ }^{16,19}$.

For cloning of the NGL-1 cytoplasmic region or its C1 and C2 fragments, we used the pEGFP-C2 vector (Clontech) double-digested with EcoRI and SalI restriction enzymes (New England Biolabs) and PCR products generated using genomic DNA as a template with the following primer pairs: NGL-1 pEGFP EcoRI forward 5'-TAGAATTCAAGATGAGGAAGCAGCACC-3' and NGL-1 pEGFP SalI reverse 5'-TAGTCGACTTAGATTTGAGTCTCTT-3'; NGL-1 C1 pEGFP EcoRI forward $5^{\prime}$-TAGAATTCAAGATGAGGAAGCAGCACC- $3^{\prime}$ and NGL-1 C1 pEGFP Sall reverse $5^{\prime}$-TAGTCGACTTACATGGGTGTGTCTCCCG-3'; NGL-1 C2 pEGFP EcoRI forward 5'-TAGAATTCGAAAGCCACCTGCCCATGC-3' and NGL-1 C2 pEGFP SalI reverse 5'-TAGTCGACTTAGATTTGAGTCTCTT-3'.

The lentiviral knockdown constructs were generated by cloning oligoduplexes into pLentiLox 3.7 (pLL 3.7) according to the protocol published online by Science gateway (http://www.sciencegateway.org/protocols/lentivirus/cloning.htm) The sh-CDKL5\#1 construct targets the region 5'-AGGAGCCTATGGAGTTGTA$3^{\prime}$, the sh-CDKL5\#2 construct is directed against the target region $5^{\prime}$ TGATAGCAGTTCTGGTACA- $3^{\prime}$ and the sh-control construct is directed against Renilla luciferase and targets the region $5^{\prime}$-GGCCTTTCACTACTCCTAC- $3^{\prime}$ pMyc-hNGL-1 was a gift from E. Kim (Korea Advanced Institute of Science and Technology, Daejeon, Republic of Korea). pEGFP-PSD95 was a gift from L. Muzio (San Raffaele Scientific Institute, Milan, Italy). pMXOCT4, pMXSOX2 and pMXKLF4 were gifts from S. Yamanaka (Gladstone Institute, University of California, USA). The S631A, S631E and S600A derivatives were generated by site-directed mutagenesis using the QuikChange II site-directed Mutagenesis Kit (Stratagene). pCAGGS-hNGL-1-iresEGFP and pCAGGS-NGL-1 ${ }^{\mathrm{S} 631 \mathrm{~A}}$-iresEGFP were generated by PCR and cloned into the EcoRI site of pCAGGS-iresEGFP. Recombinant hNGL-1 (amino acids 550-640) and hNGL-1 ${ }^{\text {S631A }}$ (amino acids 550-640) were produced from the pTYB1-NGL-1 and pTYB1-NGL-1 ${ }^{\text {S631A }}$ constructs cloned by PCR into the NdeI and XhoI sites of pTYB1 (New England Biolabs), generating a chitin-binding fusion protein. All PCR-generated constructs were verified by sequencing.

Cell culture and transfection. HEK293T, COS-7 and fibroblast cells were cultured in Dulbecco's modified Eagle's medium (Invitrogen) supplemented with $1 \%$ penicillin/streptomycin (Sigma Aldrich), $2 \mathrm{mM}$ glutamine (Sigma Aldrich) and $10 \%$ fetal bovine serum (Invitrogen) at $37^{\circ} \mathrm{C}$ with $5 \% \mathrm{CO}_{2}$. Transient DNA transfections were carried out using Lipofectamine 2000 (Invitrogen) according to the manufacturer's recommendations or the calcium phosphate precipitation method.

Primary hippocampal and cortical cultures. Primary neuronal cultures were prepared as described previously ${ }^{20}$.

Mixed-culture assay. The mixed-culture assay was carried as described previously ${ }^{40}$.

Retrovirus infection, iPSCs derivation and differentiation. Female CDKL5 fibroblasts derived from two patients' specific dermal biopsy explants were provided by T. Bienvenu's group (Cochin Institute, France). They were maintained in vitro for two passages before infection with three retrovirus cocktails (OCT4, SOX2 and KLF4). Fibroblasts $\left(2.5 \times 10^{5}\right)$ were infected with the retroviral cocktail for each reprogramming experiment; two days after infection, fibroblasts were plated on mitotically inactivated murine embryonic fibroblasts layer on Matrigel (BD)-coated dishes. After 6-8 weeks in culture in hES medium (DMEM F12, $1 \mathrm{mM}$ sodium pyruvate, $1 \times$ NEA, $20 \mu \mathrm{M} \beta$-mercaptoethanol, $20 \%$ knockout serum replacements and $10 \mathrm{ng} \mathrm{ml}^{-1}$ FGF2; Invitrogen), the first hiPSC clones appeared and were manually picked. Thus, they were cultured and expanded in human embryonic stem cell conditions on a murine embryonic fibroblast feeder layer on Matrigel (BD)-coated dishes. For differentiation into the three germ layers, hiPSC colonies were incubated in $1 \mathrm{mg} \mathrm{ml}^{-1}$ collagenase IV (Invitrogen) for $1 \mathrm{~h}$ at $37^{\circ} \mathrm{C}$, detached and collected. They were cultured in suspension for 5 days in EB medium (hES without FGF2) and plated on $1 \mathrm{mg} \mathrm{ml}^{-1}$ gelatine (Sigma)-coated dishes for up to 20 days in $20 \%$ fetal bovine serum (Invitrogen). For neuronal differentiation, embryoid bodies were cultured for five days in suspension in EB medium, and then plated and cultured on Matrigel-coated dishes in N2/FGF medium (DMEM F12, $1 \times \mathrm{N} 2$ supplement and $20 \mathrm{ng} \mathrm{ml}^{-1}$ FGF2; Invitrogen) for 3-5 days. After 3-5 days, appearing rosette-like structures were picked and manually disaggregated; then, they were plated on a layer of glial cells derived from E14.5 mouse cortices in N2/FGF medium for 1 day and then cultured in B27/AA medium (Neurobasal medium, $1 \times$ B27 supplement, Invitrogen; $20 \mu \mathrm{M}$ ascorbic acid, Fluka; $20 \mathrm{ng} \mathrm{ml}^{-1}$ BDNF,
Peprotech) for 55-60 days. For spine length analysis, manually disaggregated rosette structures were plated on Matrigel for 1 day, then infected by lentivirus carrying GFP reporter (pLL3.7-GFP) and maintained in culture for three days. Infected cells were manually detached and plated again on mouse astrocyte layers. They were cultured on astrocytes for 55-60 days in B27/AA medium and then fixed for further analysis.

Immunofluorescence microscopy. Hippocampal neurons were fixed for $10 \mathrm{~min}$ in methanol $\left(-20^{\circ} \mathrm{C}\right)$ or $4 \%$ paraformaldehyde for $20 \mathrm{~min}$ at room temperature Immunofluorescence microscopy was performed as described previously ${ }^{20}$. The following primary antibodies were used: immunopurified rabbit polyclonal antiCDKL5 (1:5; ref. 19), mouse monoclonal anti-NGL-1 (clone N49A/21; 1:500, Neuromab, NP065980), mouse monoclonal anti-GFP (clone 3E6; 1:500, Invitrogen, A11120), chicken polyclonal anti-GFP (1:2,000, Invitrogen, A10262), mouse monoclonal anti-MAP2 (clone MT-01; 1:500, Immunological Science, MAB10334), mouse monoclonal anti-PSD95 (clone K28/43; 1:200, Neuromab, P78352), mouse monoclonal anti-synaptophysin (clone 7.2; 1:1,000, Synaptic Systems, 101011), mouse monoclonal anti-VGLUT1 (clone 317G6; 1:500, Synaptic Systems, 135304), guinea-pig polyclonal anti-VGLUT1 (1:500, Synaptic Systems, 135304), mouse monoclonal anti-Pan-Shank (clone N23B/49; 1:400, Neuromab, N23B/49), mouse monoclonal anti-NR2A (clone N327/95; 1:400, Neuromab, Q00959), mouse monoclonal anti-OCT4 (1:500, Santa Cruz Biology, SC5279), rabbit polyclonal antiSOX2 (1:200, Abcam, Ab59776), mouse monoclonal anti-TRA-1-60 (clone TRA1-60; 1:200, Millipore, MAB4360), rabbit polyclonal anti-NANOG (1:200, Abcam, Ab21603) and rabbit polyclonal anti-Tuj1 (1:200, Covance). Alexa 488, 594 and 647 anti-chicken, anti-mouse, anti-rabbit and anti-guinea-pig IgG secondary antibodies (1:500, Molecular Probes) were used for detection. For alkaline phosphatase staining, cells were fixed in $2 \%$ paraformaldehyde for $10 \mathrm{~min}$, washed with PBS and incubated in $0.1 \%$ Triton X-100 NTMT $(100 \mathrm{mM} \mathrm{NaCl}, 100 \mathrm{mM}$ Tris-HCl, $100 \mathrm{mM}$ Tris and $50 \mathrm{mM} \mathrm{MgCl}_{2}$ ), supplemented with BCIP and NBT (Roche) for $10 \mathrm{~min}$. Bright-field images were obtained using an upright Leica microscope.

RNA extraction and quantitative PCR with reverse transcription. All RNA samples were extracted with an RNeasy Micro Kit (Quiagen) from 1 to $5 \times 10^{6}$ cells, according to the manufacturer's instructions. RNA strands were retrotranscribed using the Transcriptor High Fidelity cDNA Synthesis Kit (Roche) and complementary DNAs were amplified by Amplibiotherm DNA polymerase (Fisher Molecular Biology). For quantitative RT-PCR, SsoFast EvaGreen Supermix (BioRad) was used according to the manufacturer's instructions.

Immunoprecipitation and western blotting. Immunoprecipitation on cell transiently co-transfected using the calcium phosphate precipitation method or Lipofectamine 2000 (Invitrogen) and western blotting were performed as described previously $y^{20}$. The following primary antibodies were used: mouse monoclonal anti-GFP (clones 7.1 and 13.1; 1:1,000, Roche Diagnostics, 11814460001), mouse monoclonal anti-PSD95 (clone K28/43; 1:1,000, Neuromab, P78352), mouse monoclonal anti-synaptophysin (clone 7.2; 1:5,000, Synaptic Systems, 101011), mouse monoclonal anti-GAPDH (clone 6C5; 1:5,000, Millipore, MAB374), rabbit polyclonal anti-CDKL5 (1:1,000, Sigma, HPA002847), goat polyclonal antiGFP-HRP (1:5,000, Abcam), mouse monoclonal anti-V5 (1:1,000, Invitrogen, R960025) and mouse monoclonal anti-tubulin (clone DM1A; 1:15,000, Abcam, ab7291) Before CIP treatment for immunoprecipitation assays and phosphate-affinity PAGE, cells were collected and lysed in $50 \mathrm{mM}$ Tris- $\mathrm{HCl}$, at $\mathrm{pH} 8.0,150 \mathrm{mM} \mathrm{NaCl}$ and $1 \%$ NP-40 supplemented with EDTA-free protease inhibitors (Roche). For CIP treatment, beads were resuspended in $20 \mu \mathrm{l} \mathrm{H}_{2} \mathrm{O}$, split and each sample was supplied with $3 \mu \mathrm{l}$ buffer NEB3 (New England Biolabs). Then, $10 \mathrm{U}$ CIP (New England Biolabs) was added to the respective samples. All samples were incubated for $1 \mathrm{~h}$ at $37^{\circ} \mathrm{C}$ before western blot analysis.

Phosphate-affinity PAGE. The dinuclear metal complex $\mathrm{Mn}^{2+}$-Phos-tag acts as a phosphate-binding tag molecule and allows separation of phosphorylated and non-phosphorylated proteins in SDS-PAGE by mobility-shift detection. Here we used $10 \%$ SDS gels with $50 \mu \mathrm{M}$ Phos-tag acrylamide (Nard Institute) and $100 \mu \mathrm{M} \mathrm{MnCl}_{2}$ (Sigma) according to the manufacturer's recommendations.

2D-PAGE. Mouse brains were manually broken up using a dounce homogenizer and then resuspended in $500 \mu \mathrm{l}$ of lysis buffer $(5 \mathrm{M}$ urea, $2 \mathrm{M}$ thiourea, $2 \%$ CHAPS $\mathrm{w} / \mathrm{v}, 2 \%$ Zwittergent $\mathrm{v} / \mathrm{v}$ and protease and phosphatase inhibitors). The lysates were centrifuged at $16,000 \mathrm{~g}$ for $30 \mathrm{~min}$ at $15^{\circ} \mathrm{C}$ and stored at $-80^{\circ} \mathrm{C}$. The recovered supernatant was analysed to determine the total protein concentration using a BioRad protein assay and BSA as a standard. For 2D electrophoresis, $300 \mu \mathrm{g}$ total protein was loaded on each gel and each sample was run in triplicate. Before the first dimension of isoelectrofocusing, each sample was added with Destreak (final concentration: $100 \mathrm{mM}$ ) and IPG buffer (final concentration: $0.5 \%$ ) and then run 
at $50,000 \mathrm{Vh}^{-1}$ on $7 \mathrm{~cm}$ strips. The second dimension was SDS-PAGE $6 \%$, on reduction with DTE and alkylation with iodoacetamide of the strips. Resolved proteins were electrotransferred onto Hybond-ECL nitrocellulose membrane with a miniVE Blot Module (GE Healthcare) for $2 \mathrm{~h}$ at $300 \mathrm{~mA}$ in transfer buffer $(25 \mathrm{mM}$ trizma base, $40 \mathrm{mM}$ glycine, $20 \%$ methanol and $0.05 \%$ SDS). Membranes were probed for NGL-1. Detection was by enhanced chemiluminescence (ECL) reaction (ECL detection kit, GE Healthcare) and proteins were visualized on autoradiography films (Hyperfilm ECL, GE Healthcare). The lysate ( $300 \mu \mathrm{g}$ total protein), without phosphatase inhibitors, was subjected to a de-phosphorylation reaction using CIP. Membrane images were acquired with a ProXPRESS 2D Proteomic Imaging System and quantitative comparisons of spot intensities were carried out using Progenesis SameSpots (Nonlinear Dynamics).

In utero electroporation. In utero electroporation was performed as described previously ${ }^{41}$.

Immunocytochemistry for light microscopy. Immunocytochemistry on float ing $50 \mu \mathrm{m}$ brain sections, made with a CM 1850 ultraviolet cryostat (Leica), was performed as described previously ${ }^{41}$. Sections were incubated with mouse anti-GFP (clone 3E6; 1:2,000, Invitrogen, A11120) and guinea-pig anti-VGLUT1 (1:500, Synaptic Systems, 135304). For teratoma analysis, up to $2 \times 10^{6}$ iPSCs were injected subcutaneously into the dorsal flanks of nude mice. After 3 months, teratoma were explanted, fixed and dissected as described above. Sections were stained with haematoxylin and eosin for further analysis.

Dendritic protrusion analysis. Fluorescence images were acquired using a TCS SP5 laser scanning confocal (Leica) $\times 63$ oil-immersion lens as $z$ series of $15-25$ images taken at $0.3-\mu \mathrm{m}$ intervals at $1,024 \times 1,024$ pixels resolution with $1 \times$ zoom. The confocal microscope acquisition parameters were kept the same for all scans for each experiment. Each experiment was repeated at least two, mostly three, times and images were analysed in blind using Image (NIH) software. Dendritic protrusion density was measured by counting the number of dendritic protrusions on both primary and secondary dendrites and expressed as the number of dendritic protrusions per $10 \mu \mathrm{m}$ dendritic length. The dendritic protrusion length was measured by manually drawing a vertical line from the protrusion's tip to the point where it met the dendritic shaft and the head width was measured by drawing a line perpendicular to the length. The proportion of spines in each morphology category was determined for each dendrite. For each experiment, about 300-600 spines from 25-30 dendrites derived from 10-20 neurons were analysed per condition. In the figure legends, $n$ refers to the number of neurons quantified. Statistical significance was determined by Student's $t$-test.

Electrophysiology. Standard patch-clamp whole-cell recordings were taken from primary hippocampal neurons transfected at DIV7 with sh-control or sh-CDKL5\#1 and labelled for GFP at DIV14 as described above. The neurons were continuously perfused with artificial cerebrospinal fluid of the following composition: $124 \mathrm{mM}$ $\mathrm{NaCl}, 2 \mathrm{mM} \mathrm{KCl}, 3 \mathrm{mM} \mathrm{KH} \mathrm{PO}_{4}, 26 \mathrm{mM} \mathrm{NaHCO}, 1.3 \mathrm{mM} \mathrm{MgCl} 2,2.5 \mathrm{mM}$ $\mathrm{CaCl}_{2}$ and $10 \mathrm{mM}$ glucose (final pH 7.4). Recording borosilicate glass micropipettes (outside diameter $1.5 \mathrm{~mm}$, inside $0.86 \mathrm{~mm}$ ) were pulled to a resistance of 4-10 MOhms when filled with the following medium: $120 \mathrm{mM}$ potassium gluconate, $10 \mathrm{mM} \mathrm{KCl}, 3 \mathrm{mM} \mathrm{MgCl}_{2}, 10 \mathrm{mM}$ HEPES, $2 \mathrm{mM} \mathrm{MgATP}$ and $0.2 \mathrm{mM} \mathrm{NaGTP}$, at pH 7.2 (adjusted with $\mathrm{KOH}$ ), $280 \mathrm{mOsm}$. mEPSCs were observed in the presence of artificial cerebrospinal fluid containing $1 \mathrm{mM}$ tetrodotoxin and $10 \mathrm{mM}(-)$ bicuculline methiodide, using continuous $60 \mathrm{~s}$ epochs of mEPSCs recorded at $-60 \mathrm{mV}$ membrane potential with an Multiclamp 700B amplifier (Molecular Devices) with a low-pass filter of $2 \mathrm{KHz}$. Data were digitized at $2-5 \mathrm{KHz}$ with a Digidata 1322A (Molecular Devices) controlled by Clampex 9 software (Molecular
Devices) and analysed off-line with Clampfit 9 software (Molecular Devices), after processing with a band pass of $\sim 2 \mathrm{~Hz}$ to $1 \mathrm{KHz}$ using a single-pole digital filter. Event selection was visually checked for all data. The statistical analysis was performed using the paired $t$-test expressing population mean as the mean \pm s.e.m.

Recombinant protein purification. hNGL-1 and hNGL-1 ${ }^{\mathrm{S} 631 \mathrm{~A}}$ were cloned in the pTYB1 expression construct, expressed in Escherichia coli strain ER2566 and induced with $0.5 \mathrm{mM}$ isopropyl- $\beta$-D-thiogalactopyranoside (IPTG) at $30^{\circ} \mathrm{C}$ for $5 \mathrm{~h}$. Following induction, extracts were prepared by resuspending the bacteria in $750 \mathrm{mM} \mathrm{NaCl}, 20 \mathrm{mM}$ Tris- $\mathrm{HCl}$, at $\mathrm{pH} 8.0,1 \mathrm{mM}$ EDTA and $0.1 \%$ Triton $\mathrm{X}-100$ with protease inhibitors (Sigma) and sonicated. Following centrifugation at $16,000 \mathrm{~g}$ for $10 \mathrm{~min}$, the lysate was added to chitin-agarose beads (New England Biolabs). Fusion proteins were cleaved on the column overnight by incubation with lysis buffer containing $50 \mathrm{mM}$ dithiothreitol. Eluted fractions containing the bulk of NGL-1 were pooled. Recombinant purified NGL-1 and NGL-1 ${ }^{\text {S631A }}$ were dialysed for $16 \mathrm{~h}$ at $4{ }^{\circ} \mathrm{C}$ against the kinase buffer used in the phosphorylation assay.

In vitro phosphorylation assays. HEK $293 \mathrm{~T}$ cells were transfected with 6Myc-CDKL5 and 6-Myc plasmids. At $48 \mathrm{~h}$ post-transfection, transfected cells were lysed in $50 \mathrm{mM}$ Tris- $\mathrm{HCl}$, at $\mathrm{pH} 8.0,500 \mathrm{mM} \mathrm{NaCl}, 0.1 \% \mathrm{NP}-40,1 \mathrm{mM}$ dithiothreitol, phenylmethyl sulphonyl fluoride and protease and phosphatase inhibitors (Sigma). Equal amounts of protein extracts were pre-cleared for $1 \mathrm{~h}$ with $40 \mu \mathrm{l}$ of $100 \%$ mouse IgG-agarose beads at $4{ }^{\circ} \mathrm{C} ; 40 \mu \mathrm{l}$ of $100 \%$ agarose conjugate anti-c-Myc (Sigma) was added to the extracts and the mixture was incubated for $2 \mathrm{~h}$ at $4{ }^{\circ} \mathrm{C}$. Immunocomplexes were collected by centrifugation, washed 5 times and incubated with $1.5 \mu \mathrm{g}$ of recombinant protein in a kinase buffer in the presence of $7 \mu \mathrm{Ci}$ of $\left[\gamma^{-3} \mathrm{P}\right] \mathrm{ATP}$ and $25 \mu \mathrm{M}$ of unlabelled ATP and incubated for $30 \mathrm{~min}$ at $30^{\circ} \mathrm{C}$. The reaction was stopped by the addition of Laemmli buffer and the reaction mixture was directly loaded onto a $15 \%$ SDS-PAGE; ${ }^{33} \mathrm{P}$-labelled proteins were detected by autoradiography.

Pulldown. Myc-CDKL5 was first translated in vitro using the TNT Quick Coupled Transcription/Translation System (Promega) and then incubated with intein-immobilized chitin-agarose beads or with hNGL-1-intein-immobilized chitin-agarose beads for $4 \mathrm{~h}$ at $4{ }^{\circ} \mathrm{C}$. Immunocomplexes were collected by centrifugation, washed three times with $750 \mathrm{mM} \mathrm{NaCl}, 20 \mathrm{mM}$ Tris- $\mathrm{HCl}$, at $\mathrm{pH}$ 8.0, $1 \mathrm{mM}$ EDTA and $0.1 \%$ Triton X-100 with protease and phosphatase inhibitors (Sigma), separated on SDS-PAGE and subjected to western blotting with anti-Myc and anti-chitin-binding-domain antibodies.

Lentivirus and retrovirus production. VSVG-coated particles were packaged in HEK293T cells.

Synaptosome and PSD preparation. The synaptosome fraction was prepared from rat brains as described previously ${ }^{42}$. PSD fractions were prepared from the synaptosome fraction as described previously ${ }^{43}$.

Mutation search of $N G L-1$ and NGL-2. For mutation search the single coding exons of NGL-1 and NGL-2 together with their immediately flanking non-coding regions were screened for mutations in a cohort of 51 patients who had been diagnosed with RTT, infantile spasms or a variant of RTT, in whom no MECP2 or CDKL5 mutation had been identified. All blood samples were obtained after provision of informed consent. DNAs were amplified by PCR using several primer pairs. Primer sequences are available on request from V.M.K. PCR products were subjected to denaturing high-performance liquid chromatography or directly sequenced using conventional Sanger sequencing. 

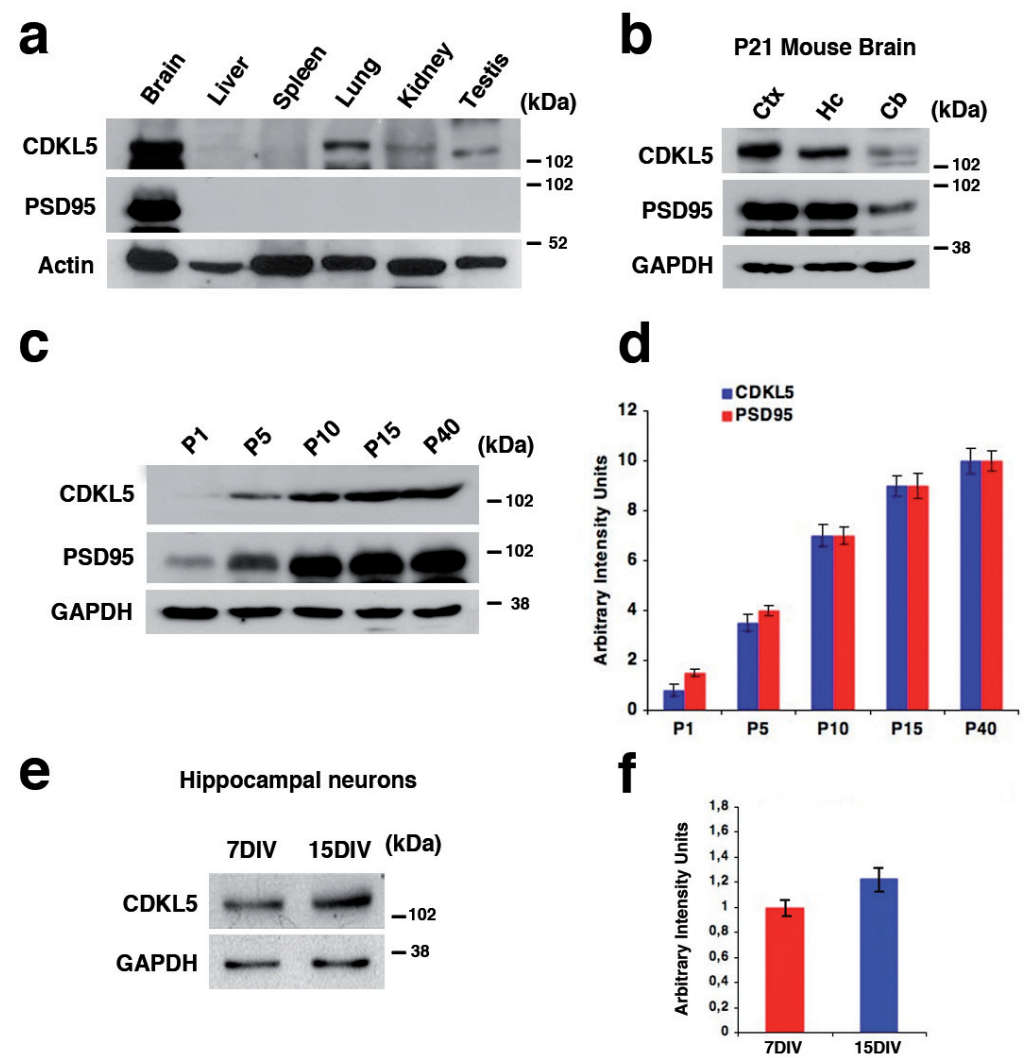

Figure S1 CDKL5 protein expression pattern and localization in mouse brain. (a) Multiple-tissue western blot from a postnatal day (P) 21 mouse probed with an antibody against CDKL5. PSD95 was visualized for comparison. Actin was visualized for normalization. (b) Widespread distribution of CDKL5 protein in postnatal day (P) 21 mouse brain regions. Ctx, cortex; $\mathrm{Hc}$, hippocampus; $\mathrm{Cb}$, cerebellum. PSD95 was visualized for comparison.
GAPDH was visualized for normalization. (c,d) Western blot analysis of CDKL5 protein expression levels in mouse brain tissues at different developmental stages. PSD95 was visualized for comparison. GAPDH was visualized for normalization. (e,f) CDKL5 protein level raises in primary hippocampal neurons cultured in vitro from DIV 7 to DIV 15. GAPDH was visualized for normalization. 


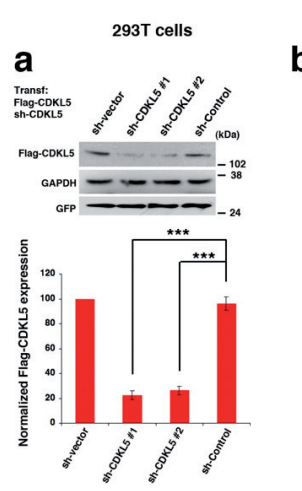

b

Rat hippocampal neurons
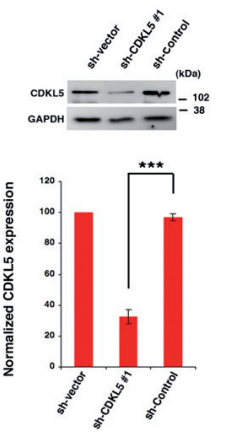

GFP-CDKL5 +
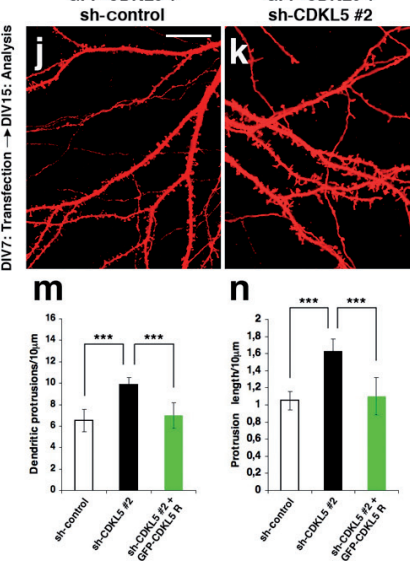

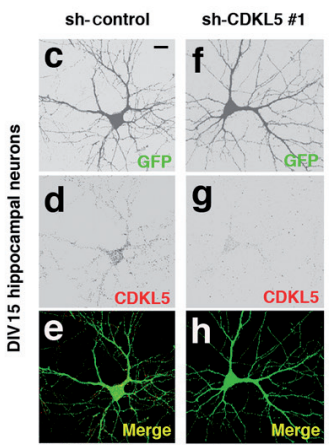

GFP-CDKL5 + GFP-CDKL5 R + sh-CDKL5 \#2 sh-CDKL5 \#2
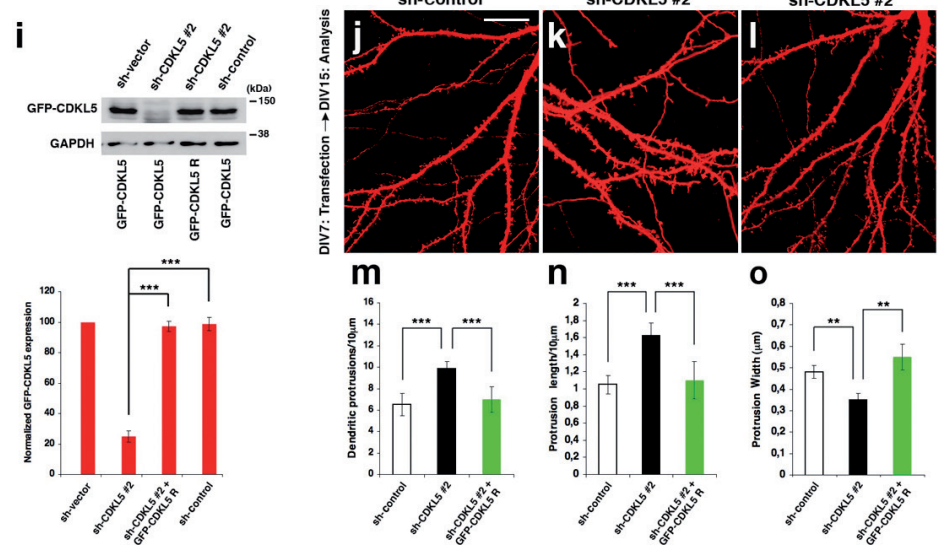

0

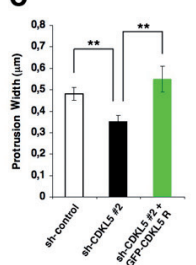

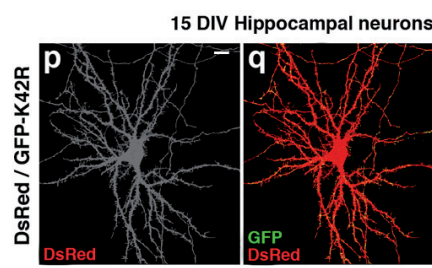

Control GFP-K42R $\mathbf{t}$ 用图

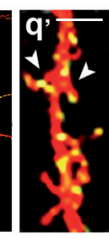

$\mathbf{u}$

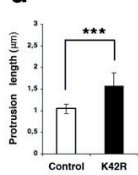

V

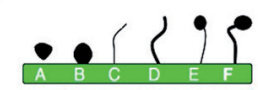

$A / B$ Stubby and mushroom-shaped spines C/B Stilopodia-like spines
E/F thin headed spines

W

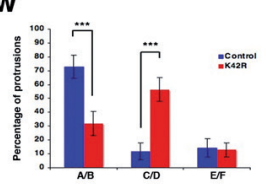

Figure S2 Knock-down of CDKL5 levels using RNAi approach. (a) Total cell lysates of HEK293T cells transfected with the indicated plasmid were subjected to immunoblot analysis using Flag and GFP antibodies. GAPDH was visualized for normalization ( $* * * p<0.001, t$ test). (b) Western blot of endogenous CDKL5 levels in DIV 15 hippocampal neurons infected at DIV 7 with sh-vector, sh-control or sh-CDKL5\#1. GAPDH was visualized for normalization ( ${ }^{* *} \mathrm{p}<0-001, \mathrm{t}$ test). (c-h) CDKL5 immunostaining in DIV 15 primary hippocampal neurons transfected with sh-control or sh-CDKL5\#1. (e,h) merged images. (i-o) Transient expression of the CDKL5 knock-down resistant form (CDKL5R). CDKL5R rescues the phenotype of CDKL5 knockdown neurons. (i) HEK293T cells were transfected with the indicated plasmids, recovered $48 \mathrm{~h}$ post-transfection and the expression levels of exogenously expressed GFP-CDKL5 were analyzed by immunoblotting with anti-GFP antibody. GAPDH was visualized for normalization. (j-I) Representative images of primary hippocampal neurons transfected at DIV 7 with the indicated plasmids and labeled for GFP at DIV 14. (m-o) Quantification of dendritic protrusion density $(\mathbf{m})$, length $(\mathbf{n})$ and head width (o) of sh-vector, sh-control and sh-CDKL5\#1 transfected neurons. Bar graphs show mean \pm s.e.m. $n=12$ neurons for sh-vector, $n=16$ neurons for sh-control and sh-CDKL5\#1 (*** $p<0.001,{ }^{* *} p<0.01$, t test). ). $n$ deriveds from three independent experiments. (p-q') Primary hippocampal neurons were transfected at DIV 7 with DsRed alone or in combination with GFP-CDKL5K42R and immunolabeled at DIV 14 for GFP and DsRed. q' shows higher magnification of q. Arrowheads in q' show a clear enrichment of the K42R immunostaining in dendritic spines. $(r, \mathbf{s})$ Representative images of single dendrites of DsRed-positive primary hippocampal neurons expressing either, control or GFP-CDKL5K42R. (t,u) Quantification of dendritic protrusion density $(\mathbf{t})$ and length $(\mathbf{u})$ of DsRed or DsRed plus GFP-CDKL5K42R transfected neurons. Bar graphs show mean \pm s.e.m. $n=15$ neurons for each condition $(* * * p<0.001, \mathrm{t}$ test $) . n$ deriveds from four independent experiments. (v) Schematic representation of dendritic protrusion morphology categories. (w) Quantification of the mean percent of spines in each morphology category in neurons expressing DsRed or DsRed plus GFP-CDKL5K42R. Bar graphs show mean \pm s.e.m. $n=15$ neurons for each condition ( ${ }^{* *} p<0.001, t$ test). $n$ deriveeds from four independent experiments. Scale bars: $10 \mu \mathrm{m}$ (c-h, j-l, p, q), $5 \mu \mathrm{m}$ (q'). 


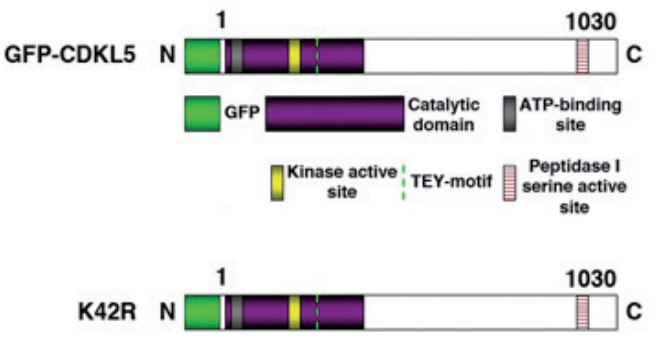

$\Delta \mathrm{N}$

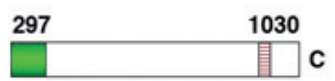

$\triangle 781 \quad$

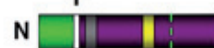

525

$\Delta 525$

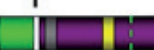

781
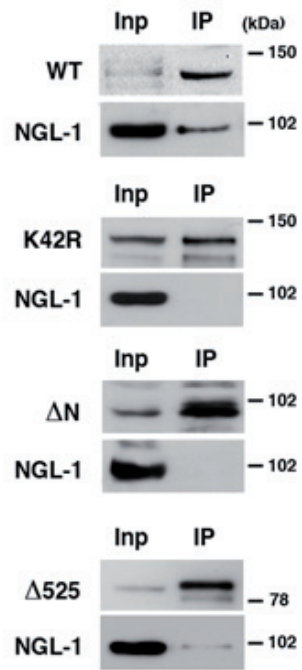

Inp IP

$\Delta 781$

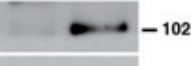

NGL-1

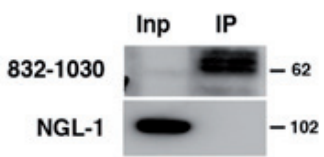

GFP

Inp IP
GFP
8321030

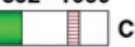

Figure S3 CDKL 5 knock-down does not affect inhibitory synapse number. (a) Primary hippocampal neurons were transfected at DIV 7 with sh-control or sh-CDKL5\# 1 and immunostained at DIV 14 with anti VGAT and gephyrin antibodies. Bar graph shows mean \pm s.e.m. $n=15$ neurons for sh-control and sh-CDKL5\#1. $n$ deriveds from three independent experiments. (b-d) CDKL5 knock-down in DIV 15 cultured neurons did not reduce the frequency (c) nor the amplitude (d) of mIPSCs. $n=5$ neurons for each condition. $n$ deriveds from three independent experiments. 
a

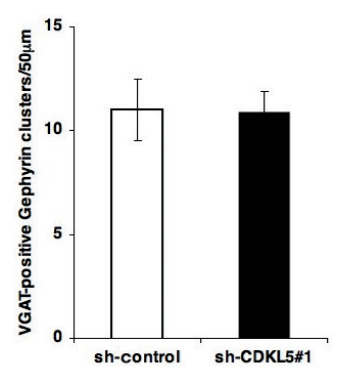

b

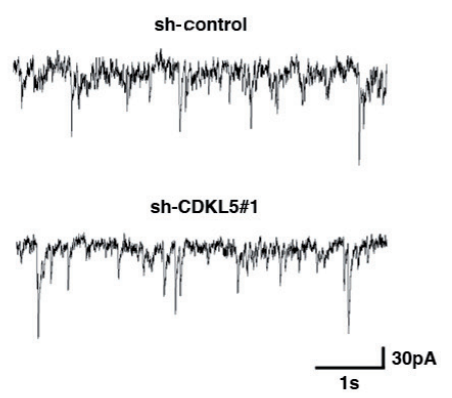

C

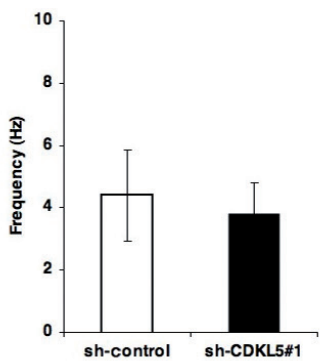

d

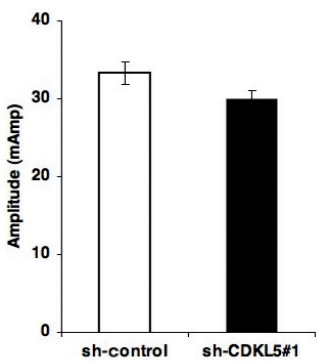

Figure S4 CDKL5 requires the catalytic domain for the interaction with NGL1. Total cell lysates of HEK293T cells double transfected with Myc-NGL-1 plus GFP-CDKL5 (WT and mutants) were subjected to immunoprecipitation with monoclonal anti-GFP antibody. Immunoprecipitated proteins as well as $10 \%$ of the cell extracts were separated by electrophoresis and analyzed by immunoblotting using antibodies indicated on the left side of each panel. 

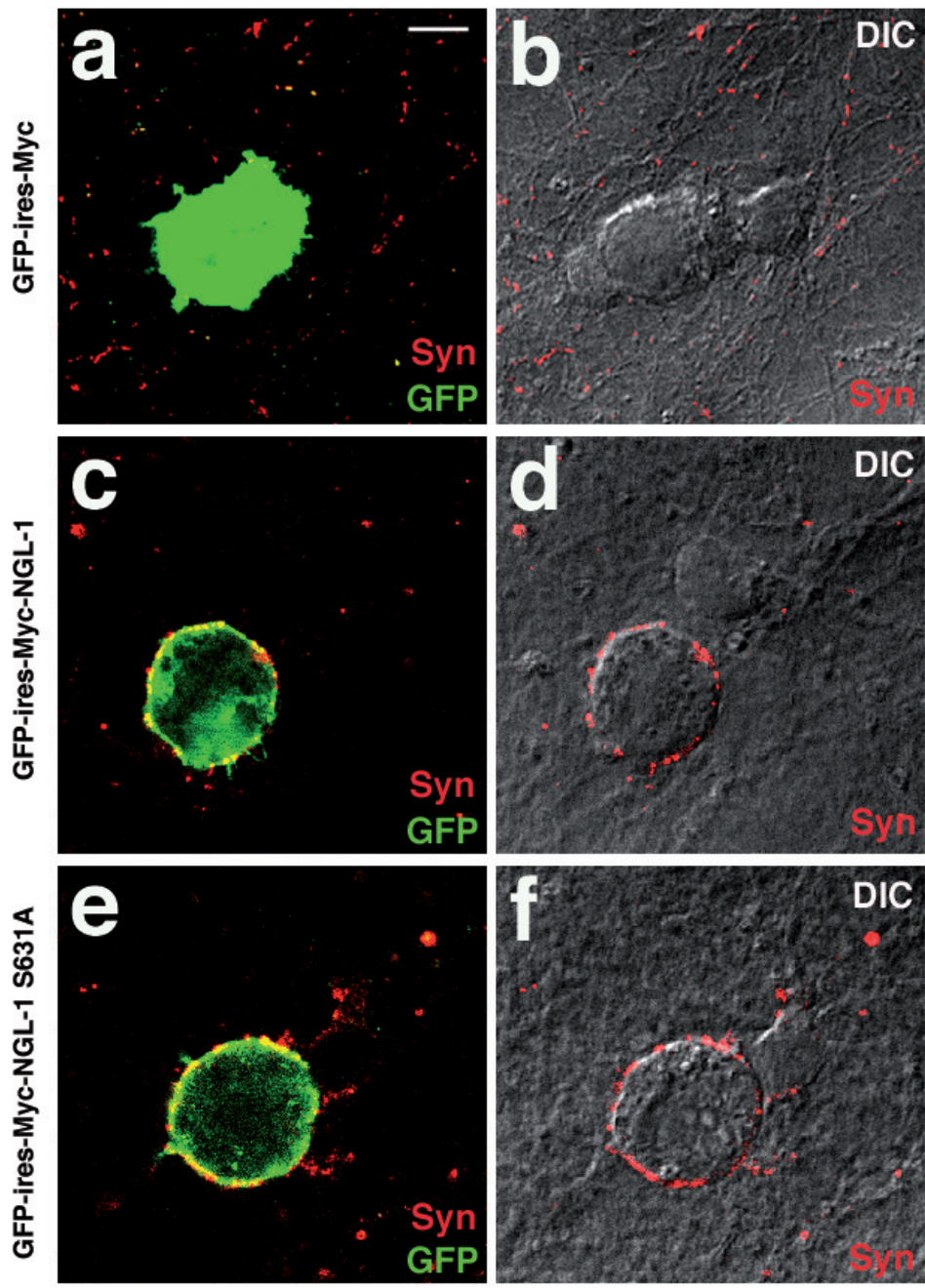

Figure S5 NGL-1 S631A induces pre-synaptic differentiation. HEK293T cells

NGL-1 S631A (e,f), were co-cultured with hippocampal neurons (DIV 9-12) transfected with GFPiresMyc (a,b), GFPiresMyc-NGL-1 (c, d) or GFPiresMycand stained for GFP and synaptophysin (Syn). Scale bars: $10 \mu \mathrm{m}$ (a-f). 
a

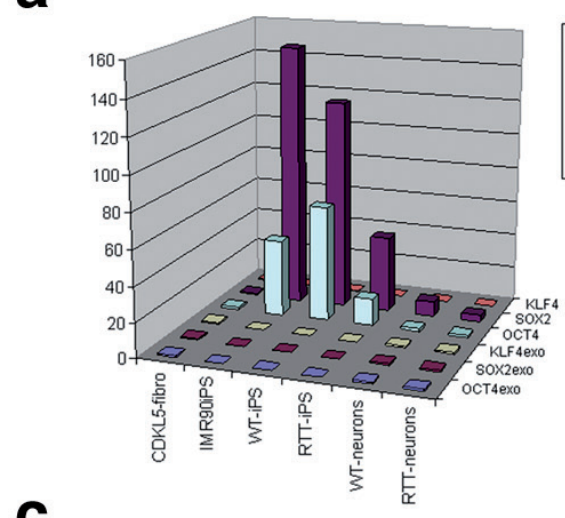

b

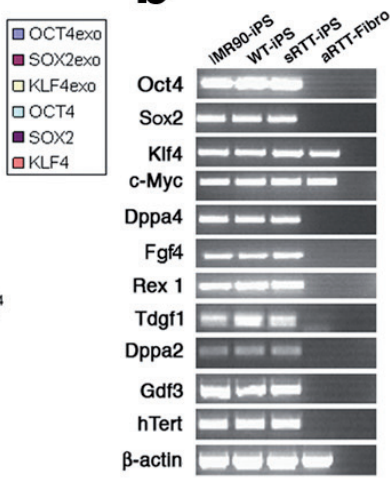

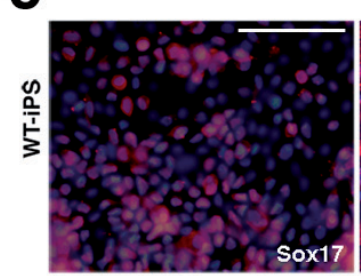
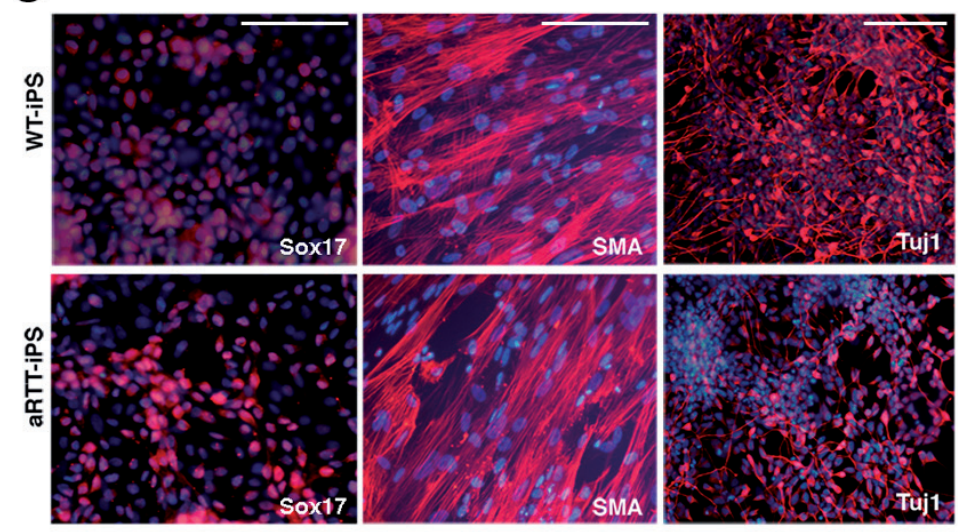

d

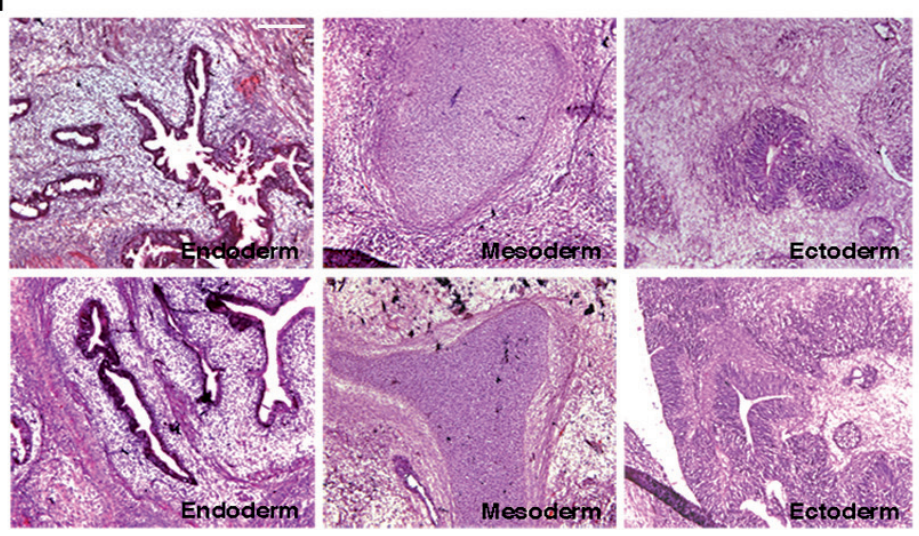

Figure S6 Generation and characterization of iPSC clones derived from CDKL5 patients. (a) iPS derived clones show complete silencing of the retroviral transgene and activation of the endogenous counterparts.

(b) RT-PCR expression analysis of a set of genes associated with pluripotency, which are activated in aRTT iPSCs. (c) Representative images of cells from the three germ layers, endoderm (Sox17+), mesoderm (SMA) and ectoderm (TuJ1) induced by iPSC in vitro

differentiation. (d) Histological sections of iPSC-derived teratomas, which display many differentiated areas with tissues of all the three germ layers. Scale bar: $100 \mu \mathrm{m}$ (c, d). 


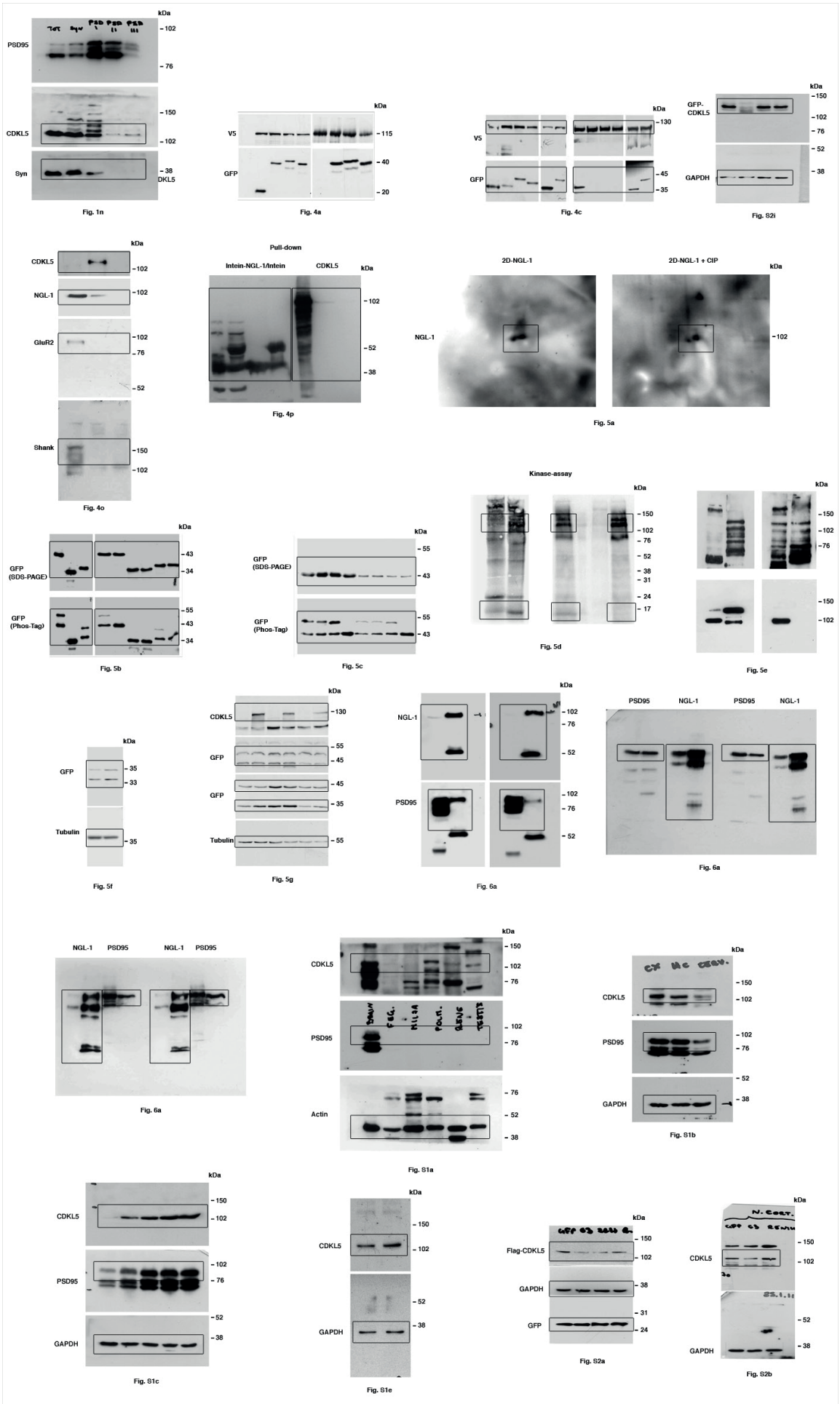

Figure S8 Full scans. 


\begin{tabular}{|c|c|c|c|c|c|c|c|c|c|}
\hline Individuals & \multicolumn{5}{|c|}{ 46, XX (R59X) } & \multicolumn{4}{|c|}{ 46, XX (L220P) } \\
\hline iPSC lines & R50X \#14 & R50X \#20 & R50X \#30 & WT \#21 & WT \#26 & L220P \#38 & L220P \#41 & WT \#34 & WT \#35 \\
\hline Tri-lineage in vitro differentiation & $\mathbf{x}$ & $\mathbf{x}$ & $\mathbf{x}$ & $\mathbf{x}$ & $\mathbf{x}$ & $\mathbf{x}$ & $\mathbf{x}$ & $\mathbf{x}$ & $\mathbf{x}$ \\
\hline Expression of viral genes & $\mathbf{x}$ & $\mathbf{x}$ & $\mathbf{x}$ & $\mathbf{x}$ & $\mathbf{x}$ & $\mathbf{x}$ & $\mathbf{x}$ & $\mathbf{x}$ & $\mathbf{x}$ \\
\hline Teratoma assays & $\mathbf{x}$ & $\mathbf{x}$ & & $\mathbf{x}$ & $\mathbf{x}$ & $\mathbf{x}$ & & $\mathbf{x}$ & $\mathbf{x}$ \\
\hline Karyotyping & $\mathbf{x}$ & $\mathbf{x}$ & $\mathbf{x}$ & $\mathbf{x}$ & $\mathbf{x}$ & $\mathbf{x}$ & $\mathbf{x}$ & $\mathbf{x}$ & $\mathbf{x}$ \\
\hline Neuronal differentiation assay & $\mathbf{x}$ & $\mathbf{x}$ & $\mathbf{x}$ & $\mathbf{x}$ & $\mathbf{x}$ & $\mathbf{x}$ & $\mathbf{x}$ & $\mathbf{x}$ & $\mathbf{x}$ \\
\hline Spine morphology & $\mathbf{x}$ & $\mathbf{x}$ & $\mathbf{x}$ & $\mathbf{x}$ & $\mathbf{x}$ & $\mathbf{x}$ & & $\mathbf{x}$ & $\mathbf{x}$ \\
\hline PSD95 puncta & $\mathbf{x}$ & $\mathbf{x}$ & & $\mathbf{x}$ & $\mathbf{x}$ & $\mathbf{x}$ & & $\mathbf{x}$ & $\mathbf{x}$ \\
\hline VGLU1 puncta & $\mathbf{x}$ & $\mathbf{x}$ & $\mathbf{x}$ & $\mathbf{x}$ & $\mathbf{x}$ & $\mathbf{x}$ & & $\mathbf{x}$ & $\mathbf{x}$ \\
\hline
\end{tabular}

